Analyzing x-ray emissions from meter-scale negative discharges in ambient air

This content has been downloaded from IOPscience. Please scroll down to see the full text.

2016 Plasma Sources Sci. Technol. 25044002

(http://iopscience.iop.org/0963-0252/25/4/044002)

View the table of contents for this issue, or go to the journal homepage for more

Download details:

IP Address: 131.155.2.68

This content was downloaded on 28/05/2016 at 12:00

Please note that terms and conditions apply. 


\title{
Analyzing x-ray emissions from meter-scale negative discharges in ambient air
}

\author{
Pavlo Kochkin ${ }^{1,2}$, Christoph Köhn ${ }^{3,4}$, Ute Ebert ${ }^{1,4}$ and Lex van Deursen ${ }^{1}$ \\ ${ }^{1}$ Department of Electrical Engineering, Eindhoven University of Technology, Eindhoven, \\ The Netherlands \\ ${ }^{2}$ Birkeland Centre for Space Science, University of Bergen, Bergen, Norway \\ ${ }^{3}$ DTU Space, National Space Institute, Technical University of Denmark, Elektrovej 328, 2800 Lyngby, \\ Denmark \\ ${ }^{4}$ Centre for Mathematics and Computer Science (CWI), PO Box 94079, NL-1090 GB Amsterdam, \\ The Netherlands \\ E-mail: p.kochkin@tue.nl and koehn@space.dtu.dk
}

Received 25 February 2016

Accepted for publication 12 April 2016

Published 27 May 2016

\begin{abstract}
When voltage pulses of $1 \mathrm{MV}$ drive meter long air discharges, short and intense bursts of $\mathrm{x}$-rays are measured. Here we develop a model for electron acceleration and subsequent photon generation within this discharge to understand these bursts. We start from the observation that the encounter of two streamers of opposite polarity launches the electrons, that they are further accelerated in the discharge field and then lose their energy, e.g., by photon emission through Bremsstrahlung. We model electron and photon dynamics in space and energy with a Monte Carlo model. Also the detector response to incoming photons is modelled in detail. The model justifies the approximation that the $\mathrm{x}$-ray bursts are isotropic in space; this assumption is used to conclude that $\mathrm{x}$-ray bursts near the high-voltage electrode with $6 \cdot 10^{4}$ photons and characteristic energies of $160 \mathrm{keV}$ closely reproduce the measured spectra and attenuation curves. The nanosecond duration of the bursts as well as their energy spectrum is consistent with model calculations.
\end{abstract}

Keywords: discharge, streamer, run-away electron, x-ray, high-voltage

(Some figures may appear in colour only in the online journal)

\section{Introduction}

\subsection{Generation of $X$ - and gamma-rays in pulsed air discharges}

The generation of x-rays in lightning was predicted in 1925 [1] and observed in impulse spark discharges in late 1960s $[2,3]$. More recently hard radiation has been detected both in thunderstorms and in MV laboratory discharges [4-15]. Microsecond fast $\mathrm{x}$-ray bursts were recently detected in an aircraft in association with lightning discharges [16]. In addition, nanosecond short electron avalanches and accompanying $\mathrm{x}$-ray emission in gas-filled diodes were investigated in Russia (see [17] and citations therein).

Current models for the high-energy radiation rely upon the energy dependence of the effective electron friction force $[18,19]$. In the rapidly developing discharge, regions of high electric fields exist near streamer or leader ionization fronts. There thermal electrons can receive a kick to overcome the friction and be accelerated in the field to high energies in a run-away process. This process occurs both in the propagation of lightning leaders and in laboratory discharges. For laboratory discharges the encounter of positive and negative steamers has been proposed as kick-off hot spot for electrons [20], which was later confirmed with nanosecond fast photographs in [14, 21]. Similar processes between leader stem and so-called space leaders might occur when negative lightning leaders propagate in a stepped manner. But direct observations of the space leader are difficult and rare [22]. Once accelerated to high energy, the electrons generate $X$ or $\gamma$-rays in collisions with air molecules through Bremsstrahlung.

In this paper we develop models to describe the $\mathrm{x}$-rays in our laboratory measurements $[15,21]$ with negative voltages 


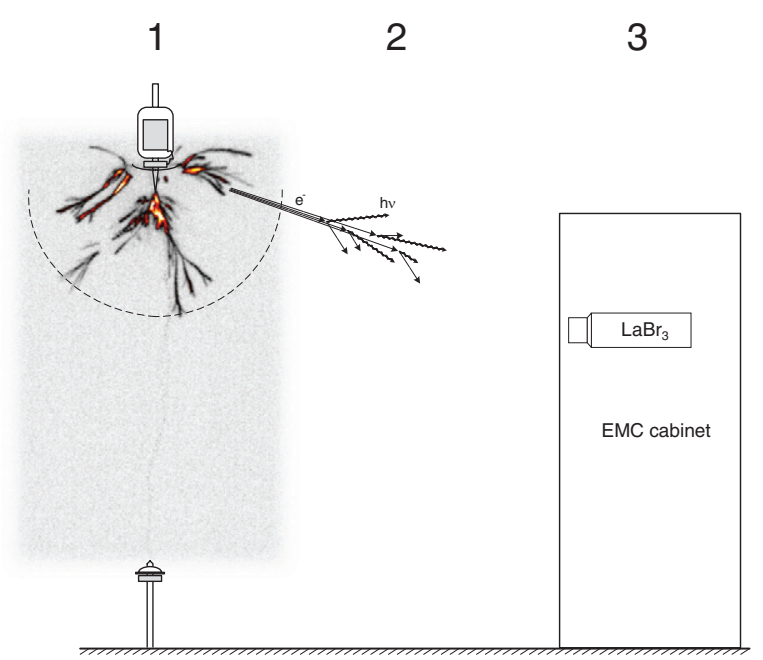

Figure 1. Sketch of the different physical regions discussed in this paper: region 1 is the interior of the discharge where the electrons are accelerated into the run-away regime. Region 2 is between discharge and detector where electron energies are maximal and most high energy photons are generated. Region 3 is the $\mathrm{x}$-ray detection facility.

of $1 \mathrm{MV}$. The advantage of a laboratory environment is that discharges can be accurately diagnosed; a disadvantage is that the setup limits the voltages to the MV range which is small compared to natural lightning. Nevertheless, discharges with up to $1 \mathrm{MV}$ are in an interesting transition regime, as the rest mass of an electron is $511 \mathrm{keV}$. As a result, above roughly $500 \mathrm{keV}$ electron energy the photons will be more relativistically beamed in the forward direction while at lower energies the photon emission is more isotropic. We include some calculation results to study the transition, although it is unlikely that $\mathrm{MeV}$ electrons occur in our experimental setup.

To understand the relation between the discharge and the measured x-ray bursts, a number of processes are investigated in this paper that are sketched in figure 1. First, there is evidence that the initial electron acceleration occurs in encounters of streamers of different polarity near the negative electrode, in region 1 in the sketch. As there are no models available yet for the full complexity of such a discharge, we assume for simplicity that electron bursts with energies in the range of 40-100 keV are emitted from this region. In section 3.1, we introduce a simplified model for the electric field in the active discharge and calculate the electron gain and loss of the electrons when traveling outward on this field. According to our estimates they can reach energies of $500-600 \mathrm{keV}$ just outside the active discharge region. Then in section 3.2 the $\mathrm{x}$-ray generating from such electrons is discussed, and in section 3.3 a Monte Carlo model for electron and photon dynamics is introduced, as previously used in [23]. In section 3.4, the electron motion in the region outside the discharge (region 2 in figure 1) is calculated, taking the electric field into account, and in section 3.5 the distribution of the generated photons in space and energy is discussed. Section 3 deals with the arrival of x-rays and fast electrons at the detector in region 3 , and with the simulation of the detector response and with the interpretation of the measurements. An important question is whether the x-ray detector signals should be attributed purely to photons, or whether high-energy electrons can reach the detectors as well. Second, to determine the x-ray generation rate of the discharges, the intensity and the spectrum, we model the detector and the absorbers when placed in front of it. These results are specific for our setup, but the applied method is general.

We focus on our laboratory measurements of negative and positive discharges with voltage pulses of $1 \mathrm{MV}$ in the wide free space of the high voltage hall. Therefore we exclude from our discussion those cases where the discharge is supported by relativistic electrons from external sources, or where the high energy photons are generated through collisions of energetic electrons with metal electrodes as in [10].

\section{Our previous observations on discharge development and $\mathrm{x}$-ray emissions}

The experiments were carried out with the 2.4 MV Marx generator at Eindhoven University of Technology. Discharges in ambient air between two conical electrodes were described in $[7,14]$ for positive voltages and in $[7,15,21]$ for negative voltages. For the sake of completeness, we briefly summarize the main aspects of the setup and we recall the essential observations needed to develop our model. The maximum applied generator voltage was $1 \mathrm{MV}$; the rise time was $1.2 \mu \mathrm{s}$. The distance between the electrodes was $1 \mathrm{~m}$, see figure 2 . The tip of the grounded electrode was at $0.5 \mathrm{~m}$ above the ground. The electric current on both electrodes and the voltage were recorded by probes connected to high-speed digital oscilloscopes. EMC cabinets assured protection against electromagnetic interference. The x-rays were registered by two fast $\mathrm{LaBr}_{3}$ scintillation detectors. The detector output is directly recorded on the digital oscilloscopes. Images of the optical emission were made by a Picos4 camera with nanosecond time resolution. Other details are provided in section 4 .

\subsection{Positive discharges}

In [14] we presented time-resolved images and showed that positive streamers continuously grow downward from the high voltage electrode (anode) while the voltage keeps rising. When the streamers approach the grounded electrode (cathode) negative counter-streamers emerge, grow upward and encounter the positive streamers. The merger of streamers and counter-streamers coincides with the maximal occurrence of x-ray bursts. Recorded photon energies are $30 \mathrm{keV}$ and higher; lower energy photons probably occur in larger number, but they are strongly absorbed in air and in the detector's aluminum casing. X-rays have never been detected before a current flows at the cathode, or before the negative counterstreamers are emitted. A fakir's bed cathode with many pins (see details in [14]) creates more negative counter-streamers, and also more x-ray bursts. We noted that the x-ray bursts coincide with high-frequency oscillations of the cathode current that are probably produced by such encounters. 


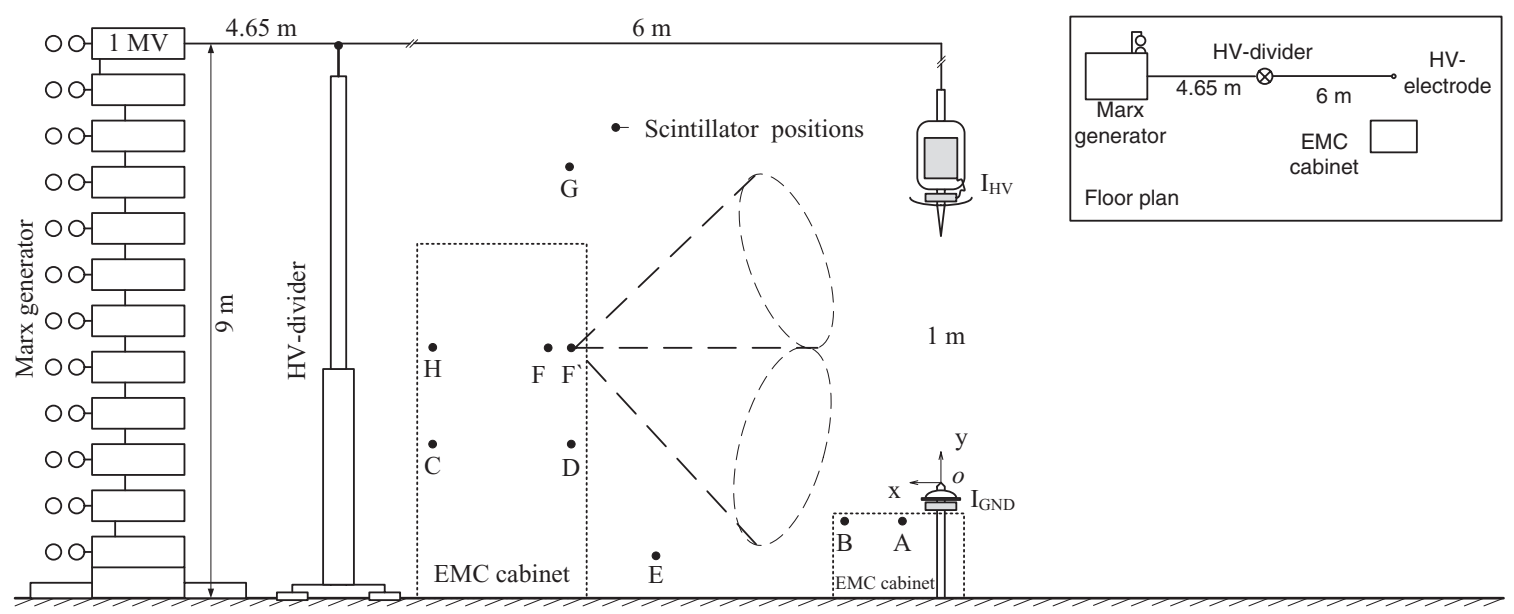

Figure 2. Sketch of the experimental set-up. The positions of the x-ray scintillator detectors are labeled from $A$ to $H$; they are all in the same vertical plane. Dashed cones indicate the detector field of view when the detector $F^{\prime}$ is placed inside a lead cylindrical collimator. The distance between the Marx generator and the spark gap is $10.65 \mathrm{~m}$. The upper right inset shows the correctly scaled floor plan. The figure is reproduced from [21].

We therefore conclude in [14] that the encounters of negative and positive streamers are a condition for the x-rays. Experiments with lead collimators demonstrate that the x-rays originate from the region close to the cathode. The anode voltage is still rising at that moment, and has a value of $0.9-1$ MV at the begin of the x-ray emission.

\subsection{Negative discharges}

The appearance of negative discharges has been discussed in [15], and their x-ray generation in [21]. In this work we analyse the x-ray source. In contrast to positive discharges, negative streamers propagate in bursts or steps that are associated with current pulses on the cathode. In each burst, the streamers in the corona propagate with a velocity of $(2.0 \pm 0.5) \times 10^{6} \mathrm{~m} \mathrm{~s}^{-1}$. The maximum extension of each burst is reasonably approximated by the instantaneous applied voltage $U(t)$ divided by the so-called stability field of $10-12 \mathrm{kV} \mathrm{cm}^{-1}$ for negative streamers, so the stepping process seems to be related to the slow rise time of $1.2 \mu \mathrm{s}$ of the voltage and the high velocity of negative streamers. We recall that negative streamers have a minimal velocity $[24,25]$. When the electrode tips are $107 \mathrm{~cm}$ apart, positive counter-streamers emerge from the grounded anode during the fourth streamer burst. Again, only then streamers of both polarities encounter and x-rays occur. However, when the separation of electrode tips is increased to $147 \mathrm{~cm}$ or larger, the peak of the x-ray emission occurs before any counterstreamers show up, and negative streamers only occasionally meet positive counter-streamers from the anode; see figure 3. $\mathrm{X}$-ray bursts have even been observed without final breakdown of the gap, which implies that the counter-streamers from the anode are not a necessary condition for x-rays. Nevertheless, x-ray bursts are always accompanied by the high-frequency cathode current oscillations. Measurements with the lead collimators again confirm that $2 / 3$ of the emission occurs near the cathode. To locate the source of the $\mathrm{x}$-ray bursts we zoomed in on the cathode region with nanosecond fast photography during the discharge development; see figure 4 . There the negative discharge develops through the formation of so-called 'pilot systems' [26] in the region pre-ionized by earlier streamers. Pilots are bipolar structures that grow in both directions-from and towards the highvoltage electrode. Again, positive and negative discharge channels (streamers) occur and encounter each other simultaneously with the peak of x-ray emission. This happens during the fourth streamer burst when the voltage has reached a value of 0.7-0.9 MV. For other interesting effects of previous discharges on streamer development, see [27, 28].

\section{Modeling the electron motion and $x$-ray generation}

\subsection{Average electron acceleration in an approximated electric field}

In both positive and negative discharges $\mathrm{x}$-rays originate in a region close to the cathode when negative and positive streamers meet nearby. That negative streamers can emit electrons that run away in the high field near the streamer head and create $\mathrm{x}$-rays by Bremsstrahlung was shown already in [29-32]. In our measurements at $147 \mathrm{~cm}$ electrode distance a space stem acts as anode. The cathode region is a dense forest of streamers and pilot systems, as shown in figure 4 . With such complexity it appears unlikely that a single electron energy distribution describes all processes involved. But only a small fraction of the electrons needs to reach high energies to explain the observed x-ray intensity. Cooray [20] suggested that the electron acceleration might be particularly efficient when a negative streamer approaches a positive one. If such process occurs near the cathode, the electron receives a kickoff with an initial kinetic energy $K_{0}$ that will increase further within the electric field in the discharge. According to [19], an initial kinetic energy of $K_{0}=32 \mathrm{keV}$ is required for electron run-away in a field of $10 \mathrm{kV} / \mathrm{cm}$, which is the stability field $E_{S}$ in a negative streamer at standard temperature and pressure (STP, $300 \mathrm{~K}$ and 1 bar). 

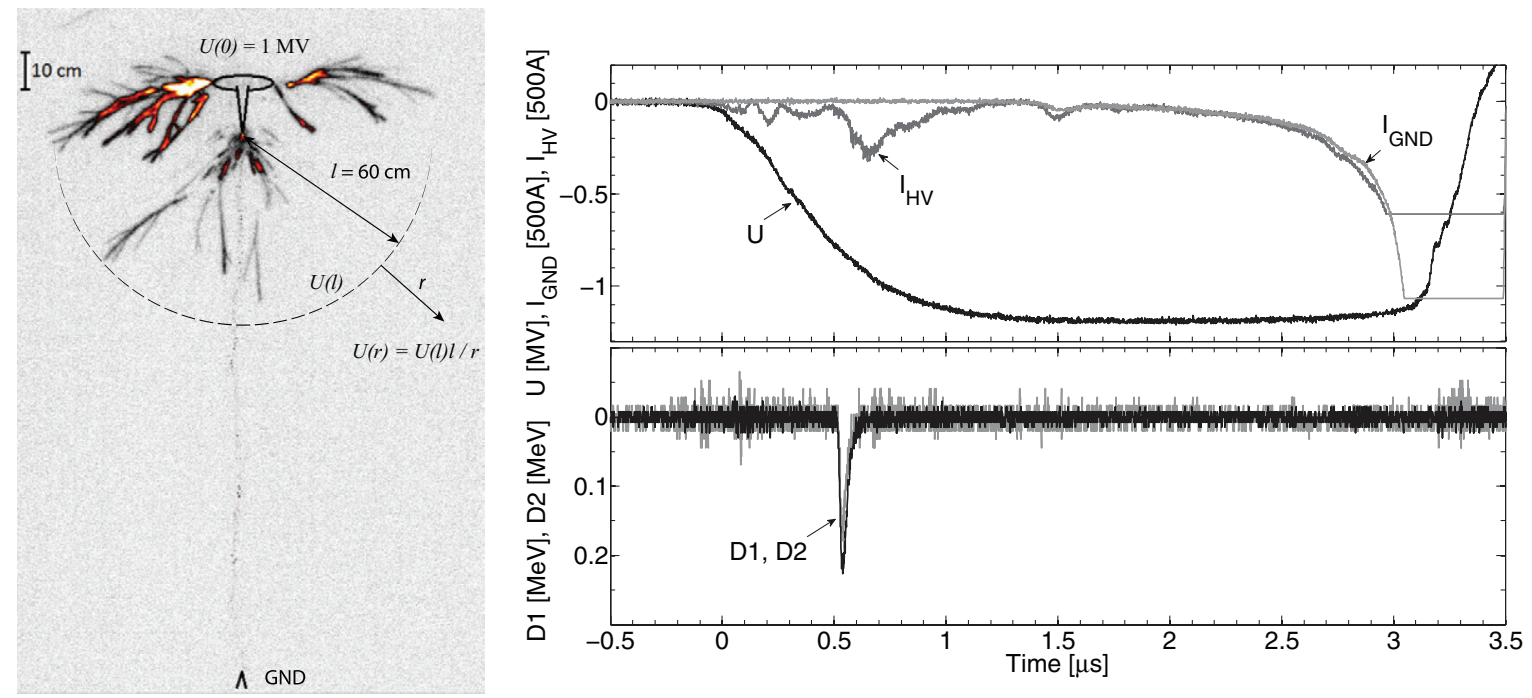

Figure 3. Left: the negative discharge at the moment of an x-ray burst, taken with an exposure time of $0.1 \mu \mathrm{s}$ from 0.45 to $0.55 \mu \mathrm{s}$; the burst happens at the middle of this time interval. A sketch of the geometry of the discharge and the electric field parameters are inserted. The faint trace between the electrodes is due to the intense light of the final arc after $3 \mu$ s that leaked through the intensifier even though it was closed. The distance between the electrode tips is $147 \mathrm{~cm}$. Right: electrical parameters of the discharge (upper panel) together with x-ray measurements (lower panel). One x-ray burst is detected by both detectors simultaneously when they were located at position $F$ next to each other. The x-rays also coincide with the onset of the fourth streamer burst, as shown by the current on the high-voltage electrode $I_{\mathrm{HV}}$.

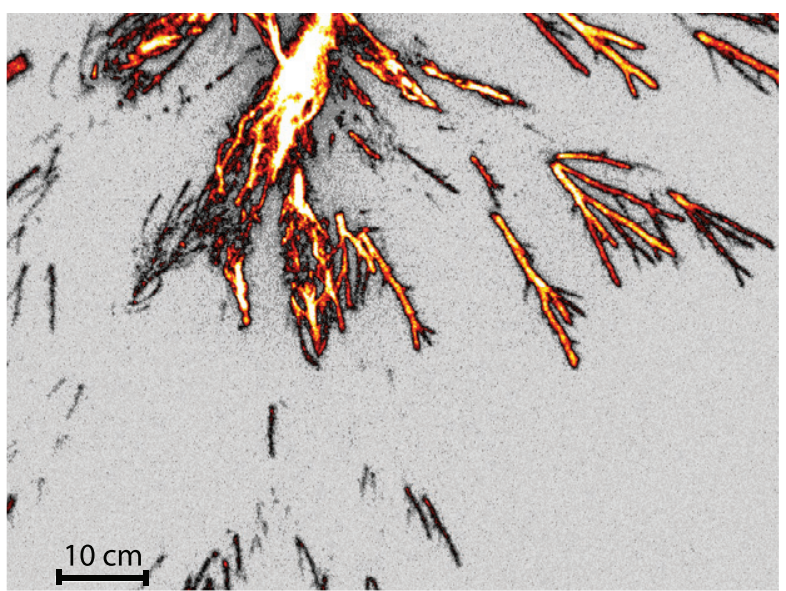

Figure 4. Zoom of the region under the cathode at $\mathrm{x}$-ray time, showing a dense bundle of streamers, counter streamers and pilot systems.

We now introduce a simplified model for the electric field distribution in the active discharge. We assume that the corona forms a sphere of radius $\ell$, as indicated in figure 3 , and that the electric field in all streamers is constant and equal to the so-called stability field $E_{S}$, and that the electric field in this region points radially outwards from the cathode located at $\mathbf{r}=(x, y, z)=0$. Because of continuity of the parallel E-field component, the corona sphere shares with the streamers a constant radial field of strength $E_{s}$ for all radii $r=|\mathbf{r}|<\ell$. When the voltage on the electrode is $U(0)<0$, one has

$$
U(\ell)=U(0)-E_{s} \ell
$$

with $E_{s}<0$ pointing inwards. Beyond $r=\ell$ there are no streamers yet, and in continued spherical approximation the electric potential outside the corona is

$$
U(r)=\frac{U(\ell) \ell}{r} .
$$

The corresponding electric field vector $\mathbf{E}(\mathbf{r})$ then reads as

$$
\mathbf{E}(\mathbf{r})= \begin{cases}E_{s} \hat{\mathbf{r}}, & r<l \\ U(l) l \hat{\mathbf{r}} / r^{2}, & r>l,\end{cases}
$$

where $\hat{\mathbf{r}}$ is the unit vector in the radial direction. This expression neglects the shape of the high voltage electrode, and should thus be considered as an approximation for small $r$. At large $r$ it does not account for the EMC cabinets, the conduction laboratory floor and the anode post that tend to enhance the field. We have $U(0)=-1 \mathrm{MV}$ and $\ell=0.6 \mathrm{~m}$ at $\mathrm{x}$-ray time, as indicated in figure 3.

The kinetic energy of electrons as a function of travelled radius $r$ can now be estimated by numerical integration of energy gain and loss over a radially outward directed electron path. The electron is kicked off near the cathode (for the sake of simplicity we assumed at $r=0$ ) with a kinetic energy $K_{0}$. For the friction force we use the NIST data [19] and for the energy gain the electric field of equation (3). The result is shown in figure 5. A tortuous electron path can be accommodated by an ad-hoc increase of the friction force, for instance by a factor of 1.2 (1.4) as shown in figure 5 by dashes (dash-dots). In this model, the maximal kinetic electron energy $K_{m}$ is reached outside the corona sphere at $r \approx 1 \mathrm{~m}$, resp. $0.9 \mathrm{~m}(0.8 \mathrm{~m})$, nearly independently of $K_{0}$. The maximal distance $R_{e}$ that the electrons can travel until all kinetic energy is exhausted, varies between 2 and $3.7 \mathrm{~m}$. Indeed, electrons may arrive at the EMC cabinet with the x-ray detectors, and even with substantial kinetic energy. The maximal kinetic energy $K_{m}$ of the electrons is about $0.5 \mathrm{MeV}$, and it is reached at about $1 \mathrm{~m}$ distance. This would be the region of maximal photon production, and also of the photons with the highest energies. 

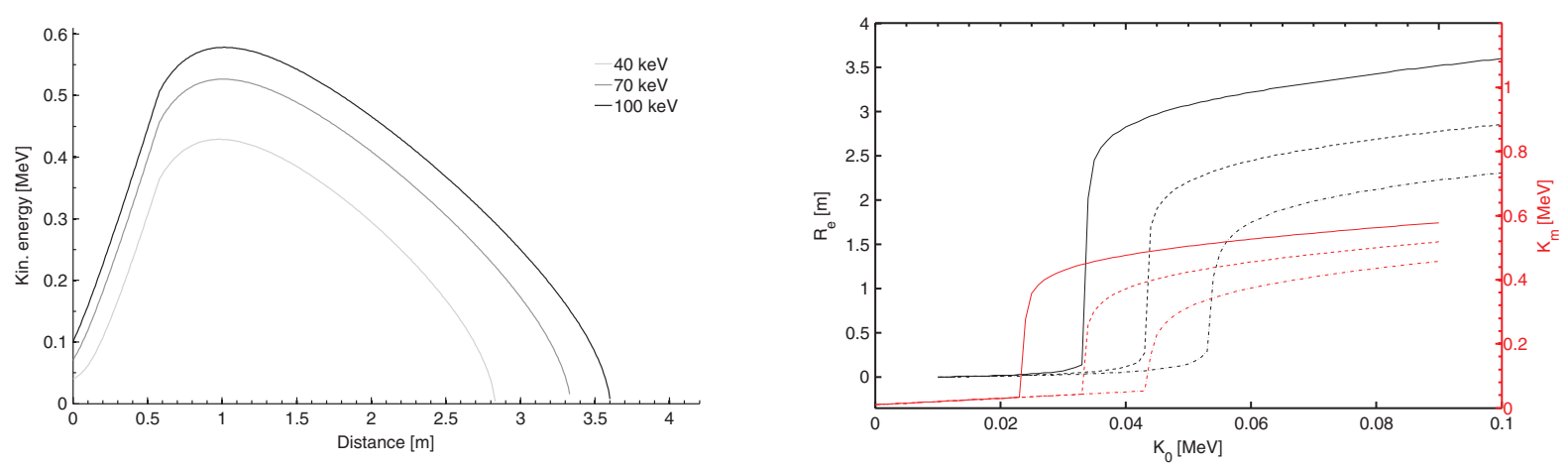

Figure 5. Left panel: Kinetic energy of electrons for starting energies $K_{0}=40,70$ and $100 \mathrm{keV}$ in the radial electric field of equation (3) as a function of distance. Right panel: average travel distance $R_{e}$ (black, left scale) until energy loss, and maximum kinetic energy $K_{m}$ (red, right scale) for electrons starting near the cathode with kinetic energy $K_{0}$ for three values of the friction force: (—) original NIST data [19], (- - -) a factor of 1.2 larger, (- - - ) a factor of 1.4 larger.

Naturally, electrons that run away at larger radii attain lower energies. In passing we note that our experiments cannot distinguish between initial electron kick-off that occurs between approaching streamers or at the tip of the resulting longer streamer.

\subsection{X-ray generation by electrons beams}

Realistic models for the complete discharge corona with streamers, counter-streamers and pilots, together with the self-generated electric field, are not available. As mentioned before, figure 4 shows an example of the streamer bundle near the cathode at x-ray time. We continue with our pragmatic approach, and start now with an electron beam with energy sufficient to generate the x-rays, and we provide approximations to determine the geometry and opening angle an x-ray burst would have. For this reason, we do not model the streamer encounter but calculate the electron motion once accelerated including the electric field outside the discharge region $r>\ell$, as described in the previous section. We consider initial electron energies above $100 \mathrm{keV}$, as electrons with lower energy are unlikely to produce photons with energies above $30 \mathrm{keV}$. Still, the lower energy electrons can liberate secondary electrons by photoionization; but again, the secondaries cannot attain sufficient energy for x-ray generation. Therefore we limit our model to electron energies between $100 \mathrm{keV}$ and 1 $\mathrm{MeV}$, the latter being the maximum any electron could possibly attain with our voltage.

Furthermore we use the fact that the fast electrons and their products do not interact, hence assuming that the space charge effects of the run-away electrons is negligible. Then the superposition principle holds which states that the products of an electron beam with given energy and angular distribution is given by the products of monoenergetic and unidirectional electron beams weighted with the energy and angular distribution of the electrons at the beam origin.

\subsection{Microscopic interactions and set-up of the Monte Carlo model}

To calculate the history of electrons and photons, we use a three dimensional Monte Carlo code as introduced in

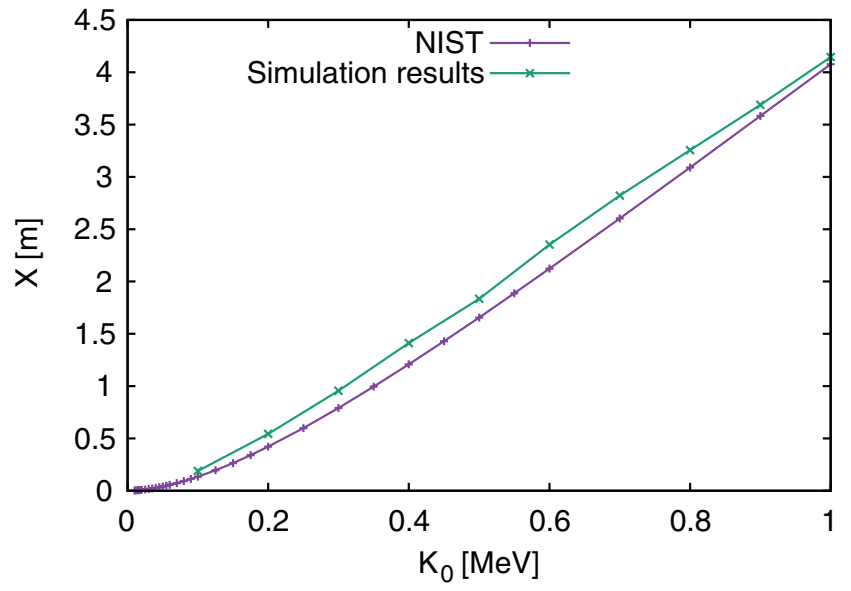

Figure 6. The simulation results and NIST data of the average path length $X$ until full energy loss of electrons for different monoenergetic electron beams with initial energies $K_{0}$.

$[23,33,34]$. The particles follow their classical or relativistic motion, and collide with a random background of air molecules at STP. As scattering processes for the electrons we include elastic electron molecule scattering [35-40], molecular excitations [35, 41], electron impact ionization [42], electron-nucleus Bremsstrahlung [43-45] and the attachment $[41,46,47]$ of electrons to oxygen, as previously used in [30, 32-34, 48-50]. Details on the implementation of the angle of elastic scattering and the energy splitting for an ionization event can be found in [33]. We note here that electron-electron Bremsstrahlung will not contribute to the photon spectrum since this process is only important for electron energies above $1 \mathrm{MeV}$ [34].

We treat the photons as particles moving with the speed of light and we include photoionization [51], Compton scattering [52, 53], pair production [45] and Rayleigh scattering [54]. The same code was used to simulate the production of photons, positrons and hadrons as well as the motion of photons and positrons in the vicinity of lightning leaders [23].

For the validation of our Monte Carlo code we simulated electron showers of 1000 electrons with initial energies of $100 \mathrm{keV}, 200 \mathrm{keV}, \ldots, 1 \mathrm{MeV}$ without electric field, and we tracked the average distance $X$ where the electrons lose all their energy; $X$ is equivalent to $R_{m}$ of section 3.1. Figure 6 

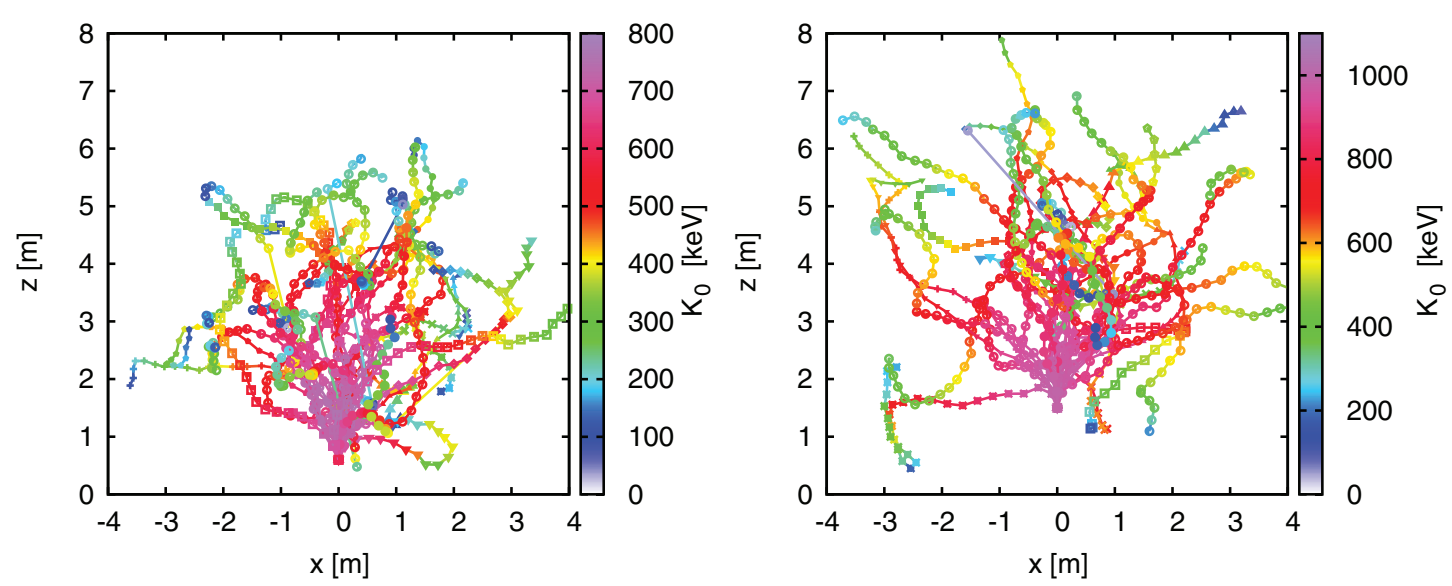

Figure 7. Left: trajectories of 50 electrons that start at $r=0.6 \mathrm{~m}$ all in the same direction radially outward with an energy $K_{0}=600 \mathrm{keV}$, projected onto the $(x, z)$ plane. The electric field $\mathbf{E}(\mathbf{r})$ is given by equation (4). Right: trajectories for $K_{0}=1 \mathrm{MeV}$ and $r=1.5 \mathrm{~m}$.

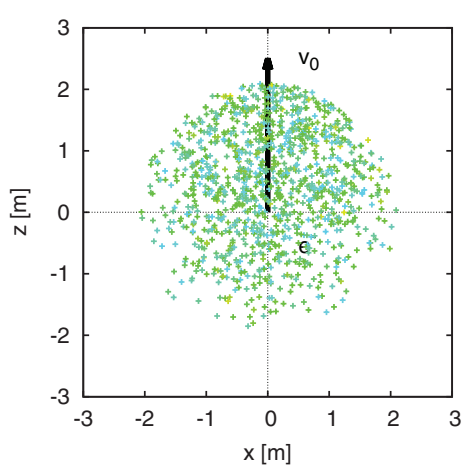

(a)

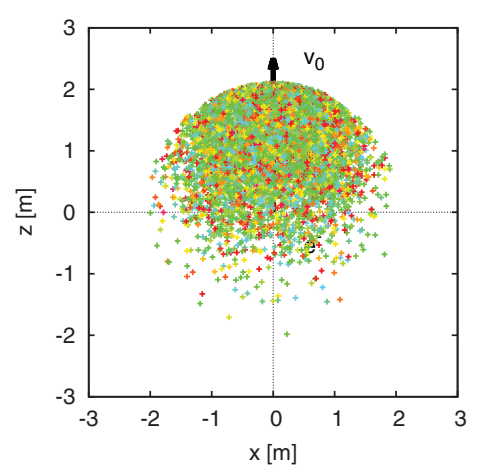

(b)

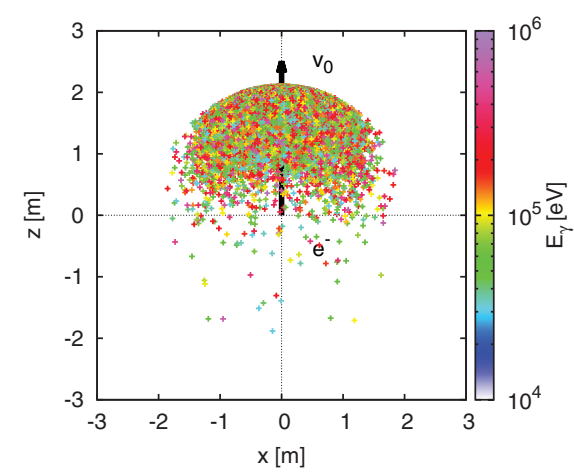

(c)

Figure 8. Position and energy of photons generated after $7 \mathrm{~ns}$ (during which time photons travel a distance of $2.1 \mathrm{~m}$ ). We start with 500000 electrons at $\mathbf{r}=\mathbf{0}$ that move in the $z$ direction (indicated by the arrow $\mathbf{v}_{0}$ ) in STP air without background electric field. The electrons initially have energy $K_{0}$. The energies $E_{\gamma}$ of the bremsstrahlung photons are color coded; only energies $E_{\gamma} \geqslant 30 \mathrm{keV}$ are included. The three panels show projections onto the transversal $x z$-plane for beams with electron energies of $K_{0}=100 \mathrm{keV}$ (a), $400 \mathrm{keV}$ (b), and $1 \mathrm{MeV}$ (c).

compares our results with the NIST data [19]. From the acceptable agreement we conclude that the code gives reliable results within the accuracy needed for our goals.

\subsection{Electron dynamics in the radial electric field}

Again, we assume that the edge of the corona is at $\ell=60 \mathrm{~cm}$ from the electrode tip, and that the voltage on the electrode has reached $U(0)=-1 \mathrm{MV}$. According to the approximation introduced in section 1.1, the radial electric field outside the streamer corona $(r>\ell)$ is

$$
\mathbf{E}(\mathbf{r})=-\frac{240 \mathrm{kV} \mathrm{m}}{r^{2}} \hat{\mathbf{r}},
$$

where $\mathbf{r}$ is the distance from the high voltage electrode. We simulate a beam of 50 electrons with initial kinetic energy $K_{0}=600 \mathrm{keV}$ on the edge of the corona at $r_{0}=0.6 \mathrm{~m}$, equivalent to $1.4 \mathrm{~m}$ from the detector at position $F$ in figure 2 . The value of the electric field is $6.7 \mathrm{kV} \mathrm{cm}^{-1}$ at $r=0.6 \mathrm{~m}$. The initial direction of the electron beams is radially outward. The left panel of figure 7 shows the trajectory of all electrons projected onto the $(x, z)$ plane.

Some electrons first gain energy up to about $800 \mathrm{keV}$ before the friction force takes over. The calculation stopped at
$30 \mathrm{keV}$ as this is the detection limit of our detectors. The right panel show similar results for electrons that start with $K_{0}=1$ $\mathrm{MeV}$ at $r=1.5 \mathrm{~m}$. For both values of $K_{0}$ many electrons travel more than 2 meter in the $r$-direction; these can reach the EMC cabinet with the detectors. At $r=2 \mathrm{~m}$ the $600 \mathrm{keV}$ beam spreads over approximately $35^{\circ}$, hence the beam is slightly focused in the initial direction. For $1 \mathrm{MeV}$ we find spreading over $20^{\circ}$.

\subsection{Photon distribution}

We calculated the Monte Carlo history of a beam of 500000 electrons injected in a region at STP but without electric field. The injection point is the origin of the coordinate system, and the direction is taken along the $z$-axis as indicated by the arrows $\mathbf{v}_{0}$ in figure 8 . The dots show the distribution of the resulting photons after $7 \mathrm{~ns}$, where we projected the positions onto the $x z$-plane and applied color coding for the photon energy $E_{\gamma}$. Only detectable photons with $E_{\gamma}>30 \mathrm{keV}$ are included. The initial electron energy $K_{0}$ is $100 \mathrm{keV}$ in panel (a), $400 \mathrm{keV}$ in panel (b), and $1 \mathrm{MeV}$ in panel (c). Clearly, the photons are rather isotropically distributed in panel (a), and increasingly beamed in panels (b) and (c). Most of the energetic photons are produced immediately after injection. 

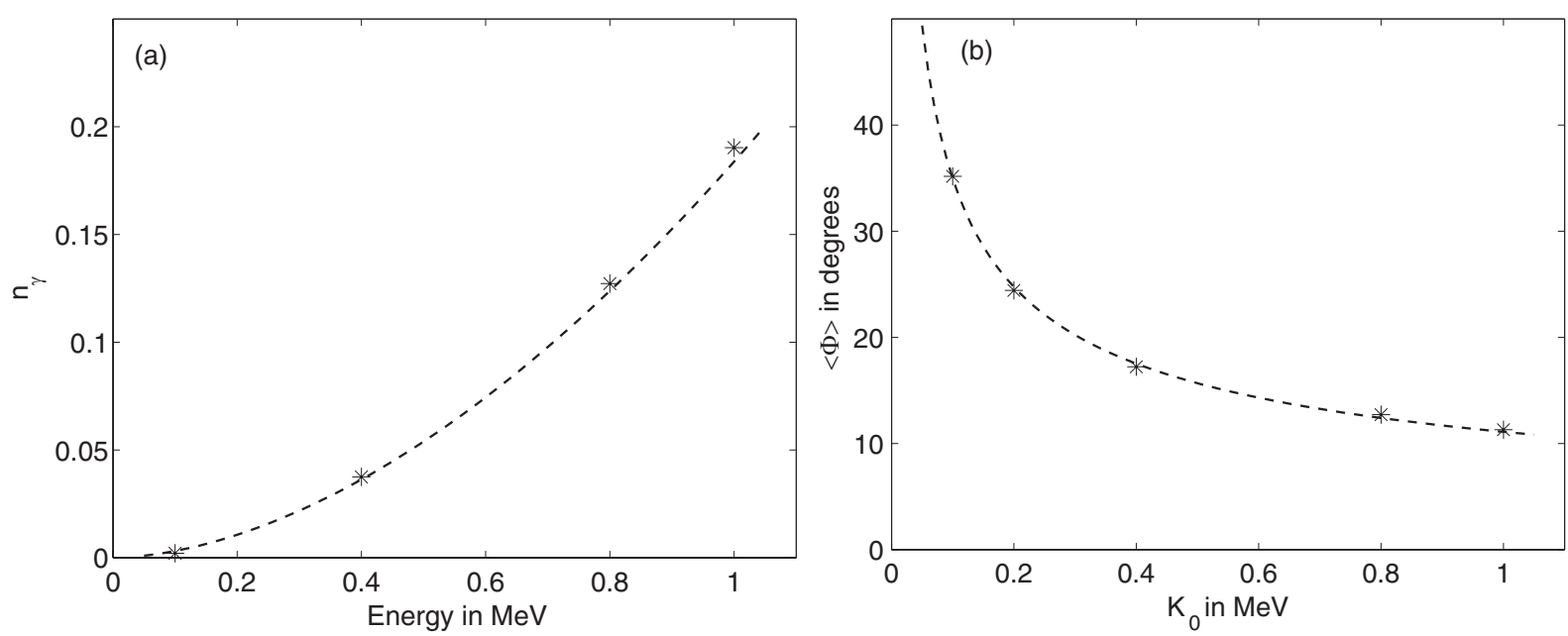

Figure 9. Properties of the burst of photons with energies $E_{\gamma} \geqslant 30 \mathrm{keV}$ as a function of the energy $K_{0}=100, \ldots, 1000 \mathrm{keV}$ of the electrons in the initial beam, evaluated with beams of 500000 electrons each, without field. (a) The number $n_{\gamma}$ of photons, expressed as fraction per electron. The dashes show the fit equation (5). (b) The average opening angle $\langle\Theta\rangle$ of the photon beam for different threshold energies. The dashes show fit equation (6).

Table 1. The Monte Carlo derived number of photons $n_{\gamma}$ for three thresholds of $E_{\gamma}$ derived from 500000 electrons with kinetic energy $K_{0}$ in STP air.

\begin{tabular}{llllll}
\hline$K_{0}(\mathrm{MeV})$ & 0.1 & 0.2 & 0.4 & 0.8 & 1 \\
\hline$E_{\gamma}>30 \mathrm{keV}$ & 1033 & 5043 & 18742 & 63588 & 95125 \\
Equation $(5)$ & $1.56 \cdot 10^{3}$ & $5.3 \cdot 10^{3}$ & $18.1 \cdot 10^{3}$ & $62 \cdot 10^{3}$ & $92 \cdot 10^{3}$ \\
$E_{\gamma}>50 \mathrm{keV}$ & 330 & 2583 & 11568 & 44433 & 69063 \\
$E_{\gamma}>100 \mathrm{keV}$ & - & 513 & 4542 & 23702 & 39324 \\
\hline
\end{tabular}

The broad distribution of positions in the figure arises from the projection, and many of the photons are found close to a sphere of radius $2.1 \mathrm{~m}$ around the origin. The increasing photon anisotropy is a relativistic effect, that becomes more pronounced at electron kinetic energies larger than the electron rest mass energy of $511 \mathrm{keV}$ [45]. Naturally, the number of energetic photons rises sharply with increasing $K_{0}$.

Figure 9 characterizes the bursts of photons with $E_{\gamma}>30 \mathrm{keV}$ further for electron beams with $K_{0}$ between $100 \mathrm{keV}$ and $1 \mathrm{MeV}$. Panel (a) shows the fraction $n_{\gamma}$ of generated photons above $30 \mathrm{keV}$ per incident electron as a function of electron energy. The dashed line is a tentative exponential fit, obtained by the nonlinear least-squares MarquardtLevenberg algorithm and omitting the uncertainty in the base,

$$
n_{\gamma}\left(E_{0}\right)=0.184 \times\left(\frac{K_{0}}{1 \mathrm{MeV}}\right)^{1.77 \pm 0.03},
$$

valid over the $K_{0}$ range mentioned. On average to generate one photon with $E_{\gamma}>30 \mathrm{keV}$ requires 94 electrons with $K_{0}=200$ $\mathrm{keV}$ or 5 electrons with $K_{0}=1 \mathrm{MeV}$. Table 1 summarizes the Monte Carlo results and the numerical values of the fit where we deliberately limited the number of digits in the results of equation (5). The largest relative deviation by a factor of 1.5 occurs for the lowest energy $K_{0}=0.1 \mathrm{MeV}$. Panel (b) in figure 9 shows the average angle $\langle\Theta\rangle=1 / N \sum_{i=1}^{N} \Theta_{i}$ where $\Theta_{i}$ is the angle of the $i$ th photon relative to the direction of the initial electron beam. The count $i=1 \cdots N$ has been taken
Table 2. The Monte Carlo derived average angle $\langle\Theta\rangle$ of photon emission for three energy thresholds $E_{\gamma}$, produced by 500000 electrons with kinetic energy $E_{0}$ in STP air.

\begin{tabular}{llllll}
\hline$E_{0}(\mathrm{MeV})$ & 0.1 & 0.2 & 0.4 & 0.8 & 1 \\
\hline$E_{\gamma}>30 \mathrm{keV}$ & 35.2 & 24.4 & 17.2 & 12.7 & 11.3 \\
Equation (6) & 35.0 & 24.7 & 17.5 & 12.4 & 11.1 \\
$E_{\gamma}>50 \mathrm{keV}$ & 34.1 & 24.0 & 17.4 & 12.5 & 11.2 \\
$E_{\gamma}>100 \mathrm{keV}$ & - & 24.5 & 17.0 & 12.4 & 11.1 \\
\hline
\end{tabular}

over three ranges of photon energy $E_{\gamma}$, either above 30 , or 50 , or $100 \mathrm{keV}$. The small differences between $\langle\Theta\rangle$ values for the various $E_{\gamma}$ thresholds indicate a major contribution by the higher energy photons. From figure 8(a) one expects an isotropic photon distribution for lower $K_{0}$, or $\langle\Theta\rangle$ approaching $90^{\circ}$. It turns out that the $\langle\Theta\rangle$ data for $E_{\gamma}>30 \mathrm{keV}$ can be approximated by the power law expression in $K_{0}$

$$
\langle\Theta\rangle=(11.10 \pm 0.63)^{\circ} \cdot\left(\frac{K_{0}}{1 \mathrm{MeV}}\right)^{-0.498 \pm 0.031},
$$

where $K_{0}$ is expressed in $\mathrm{MeV}$, with the $95 \%$ confidence interval indicated. The fit clearly shows the expected leveling of $\langle\Theta\rangle$ at $K_{0}>0.5 \mathrm{MeV}$. Anyway, the validity of the fit is strictly limited to $K_{0}>0.1 \mathrm{MeV}$ since it is not bounded for $K_{0}$ towards zero. For the sake of completeness we provide the Monte Carlo numerical data in table 2. The underlying theoretical description still has to be developed.

Figure 10 shows the energy distribution of photons generated by beams of 500000 electrons with energies $K_{0}=$ $100 \mathrm{keV}$ in panel (a), $400 \mathrm{keV}$ in panel (b) and $1 \mathrm{MeV}$ in panel(c). In order to have an acceptable scale at high energy we present $\mathrm{d} n_{\gamma} / \mathrm{d} \ln E_{\gamma}$ rather than $\mathrm{d} n_{\gamma} / \mathrm{d} E_{\gamma}$. The dots indicate the time integrated energy distributions right at the moment when each photon is generated, while the stars indicate the distributions after approximately $7 \mathrm{~ns}$ when the photons have moved for about $2 \mathrm{~m}$. Clearly, the photon distribution is the same for dots and stars at $E_{\gamma}>10 \mathrm{keV}$; such photons are hardly absorbed 


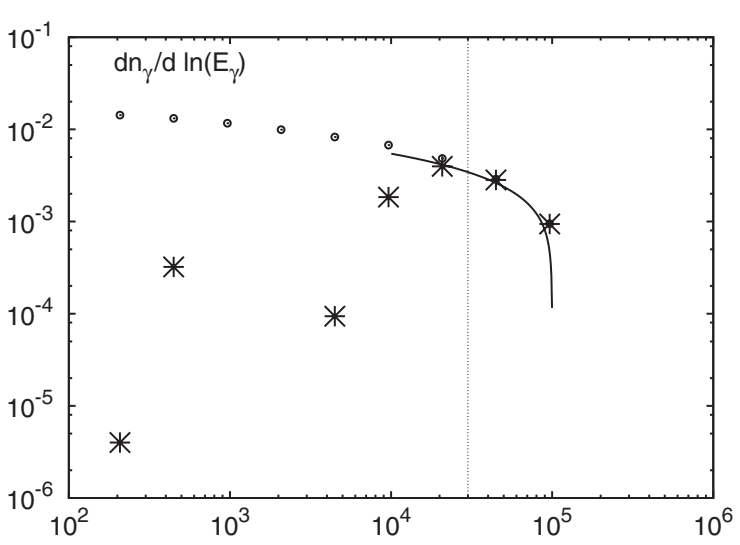

(a)

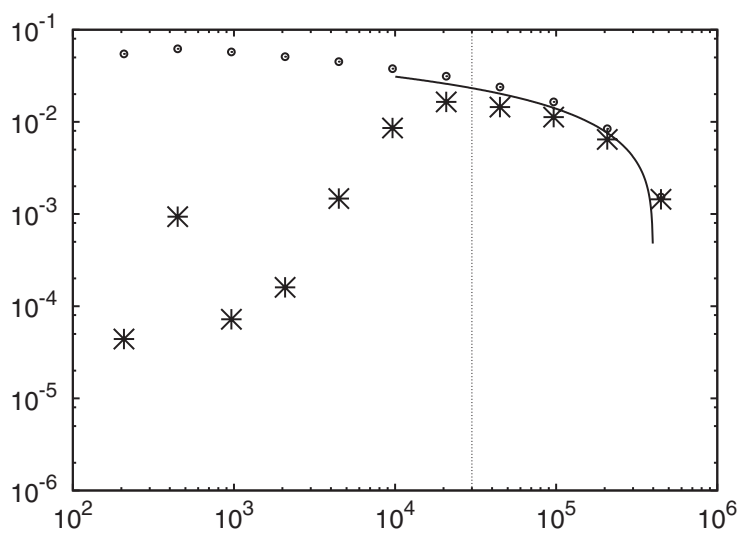

(b)

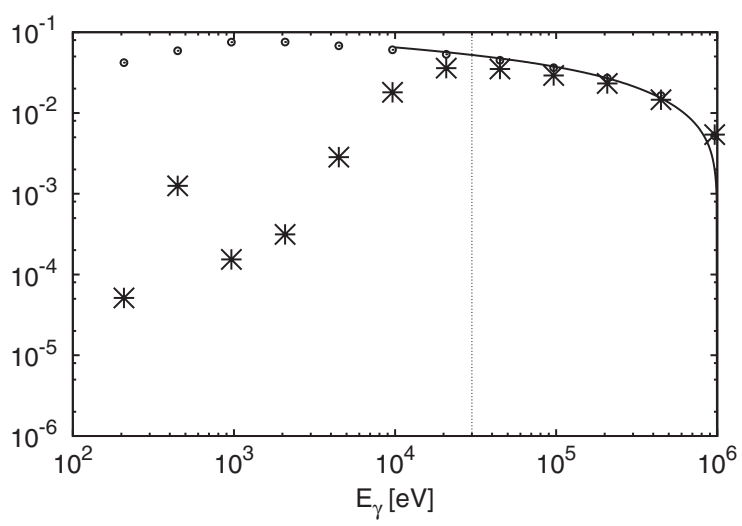

(c)

Figure 10. The energy distribution $d n_{\gamma} / d \ln E_{\gamma}$ of the photons produced by Bremsstrahlung by an electron beam with (a) $K_{0}=100$ $\mathrm{keV}$, (b) $K_{0}=400 \mathrm{keV}$, (c) $K_{0}=1 \mathrm{MeV}$, without field. The stars show the distribution after approximately $7 \mathrm{~ns}$ (which corresponds to $2 \mathrm{~m}$ travel distance). The circles show the distribution of all photons at the moment when they are produced, thus without later energy losses. The solid lines show fit (7). The dotted line shows the energy threshold of $30 \mathrm{keV}$.

when they travel $2 \mathrm{~m}$ through STP air. In contrast, photons with energies between $100 \mathrm{eV}$ and $10 \mathrm{keV}$ are mostly lost in 7 ns because of photoionization. This mechanism creates a maximum in the distribution at $E_{\gamma}$ between 20 and $30 \mathrm{keV}$ after $2 \mathrm{~m}$ in STP air.
The solid lines in figure 10 show the curve

$$
\frac{\mathrm{d} n_{\gamma}}{\mathrm{d} \ln E_{\gamma}} \sim E_{\gamma} \frac{\mathrm{d} \sigma}{\mathrm{d} E_{\gamma}}\left(K_{0}, E_{\gamma}\right) / \int_{K_{\min }}^{K_{0}} \mathrm{~d} E_{\gamma} \frac{\mathrm{d}}{\sigma} \mathrm{d} E_{\gamma},
$$

where $\mathrm{d} \sigma / \mathrm{d} E_{\gamma}$ is the Bethe Heitler cross section for photon production differential in $E_{\gamma}[43,44]$ and $K_{\min }$ is chosen as the maximum of the distribution which lies at $20 \mathrm{keV}$. The integral in the denominator normalizes the right hand side for different electron energies $K_{0}$. Photons with energies comparable to the initial electron energies are produced before electrons lose energy through other scattering processes. Thus the Bethe Heitler cross section energy is mainly useful to describe the high-energy tail of the photon energy distribution. The electrons also produce low-energy photons after they have already scattered and lost energy. But equation (7) does not include multiple scattering and thereby does not suit to describe the photon distribution at low energy, e.g., below $30 \mathrm{keV}$.

\section{Detector modelling and interpretation of the measurements}

A number of questions arise on how to correctly interpret the striking features of the x-ray measurements on long laboratory sparks: the timing and nanosecond short duration of the bursts, the spatial distribution of photons per burst, the spatial distribution averaged over many discharges, the energy distribution and the influence of photon pile-up in the detector on this energy distribution. In this section we address these questions, aided by the results of the modelling described in the previous one. We begin with a discussion of a detailed Geant4 model of the $\mathrm{LaBr}_{3}\left(\mathrm{Ce}^{+}\right)$scintillation detector. The model was verified in the laboratory by measurements of the spectra and attenuation curves for different well known radiation sources. The response of real and modeled detectors were in good agreement. The model is then available for the interpretation of the measurements.

\subsection{X-ray detectors}

Two $\mathrm{LaBr}_{3}\left(\mathrm{Ce}^{+}\right)$scintillator detectors manufactured by Saint-Gobain were mounted in EMC-cabinets and recorded the x-rays. The scintillator is a cylinder of $38 \mathrm{~mm}$ in diameter and height, with one flat surface connected to a special Hamamatsu photomultiplier with a dynode divider adapted for high speed photon counting. The scintillator has a fast rise/ decay time of $11 / 16 \mathrm{~ns}$. The output of the photomultiplier is recorded directly on the digital oscilloscope without the waveshaping electronics usually employed in photon counting. This allows to distinguish individual pulses even when pile-up occurs within the decay time of the scintillator. The linearity of the detectors was tested on radiation from ${ }^{241} \mathrm{Am},{ }^{137} \mathrm{Cs}$, ${ }^{60} \mathrm{Co}$ and remains perfect up to $2.5 \mathrm{MeV}$, which is the total absorbed energy from two gamma quanta of a ${ }^{60} \mathrm{Co}$ source [55]. The high quality EMC cabinet blocks interference on the photomuliplier output caused by the high-voltage discharge. In many discharges no X-rays occurred and only the oscilloscope input noise was recorded. In some measurement series 


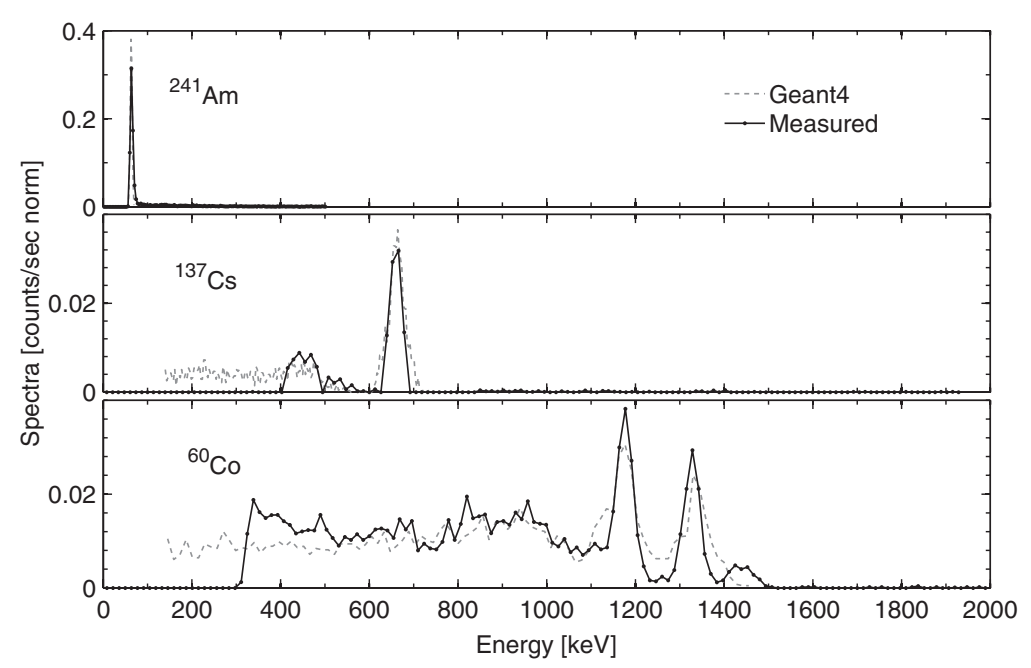

Figure 11. Comparison of measured and simulated spectra of ${ }^{241} \mathrm{Am},{ }^{137} \mathrm{Cs}$ and ${ }^{60} \mathrm{Co}$ radiation sources. Photo peaks and Compton edges are visible. Background radiation is subtracted from the measured spectra.

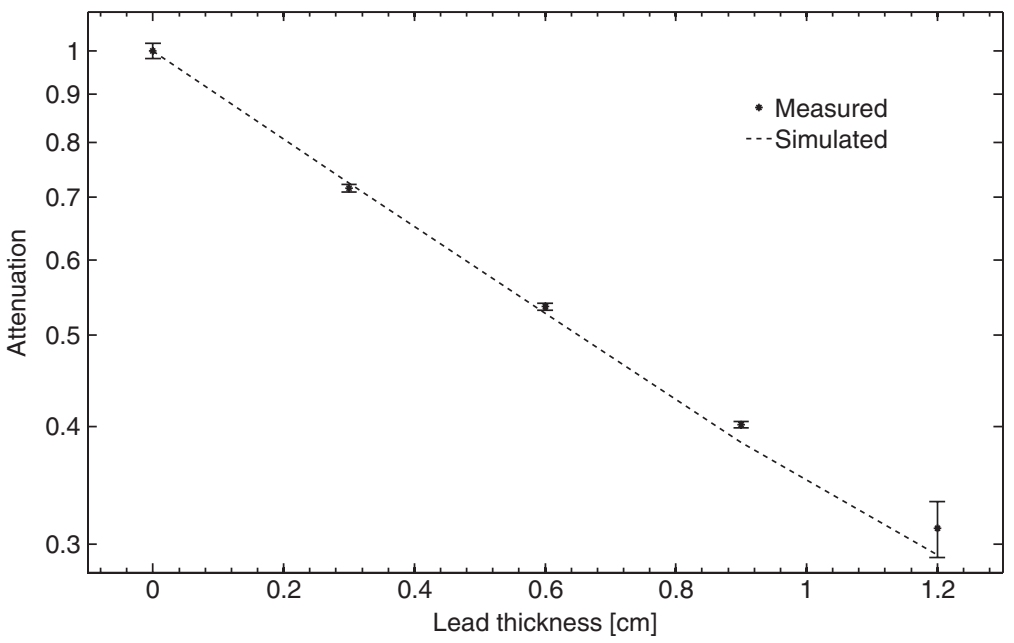

Figure 12. Comparison of measured and simulated attenuation curves. The $\mathrm{LaBr}_{3}$ detector is placed in a lead tube, its face covered by lead absorbers of different thickness. The ${ }^{137} \mathrm{Cs}$ radiation source is located at $25 \mathrm{~cm}$ distance from the detector. The same procedure was performed in Geant4. Measured and simulated attenuation curves show good agreement.

one detector was located inside a separate small EMC-cabinet as indicated in figure 2 . The total aluminum thickness between the scintillator and surrounding area is $550 \mu \mathrm{m}$ - equivalent to 1/e photon absorption at $37 \mathrm{keV}$.

\subsection{The modelling of the detector response}

A detailed Geant 4 model of a $\mathrm{LaBr}_{3}$ detector was created for further analysis. The model was validated by the comparison of the calculated and measured response on three radiation sources${ }^{241} \mathrm{Am},{ }^{137} \mathrm{Cs}$ and ${ }^{60} \mathrm{Co}$. The sources were placed at $25 \mathrm{~cm}$ distance from the detector. Figure 11 shows the comparison between measured and simulated spectra. The background was subtracted from the measured values. The rapid cut-off at low energies in measured Cs and Co spectra is caused by high trigger level of oscilloscope. The photo-peaks are well visible together with the Compton edge and Compton spectrum. The measured spectra agree well with those calculated from the model.

The attenuation curves have been measured using lead absorbers of different thickness. The $\mathrm{LaBr}_{3}$ detector was placed in a $1.5 \mathrm{~cm}$ thick lead tube and its face was covered by lead absorbers of different thickness. The ${ }^{137} \mathrm{Cs}$ radiation source was placed at $25 \mathrm{~cm}$ distance from the detector. The Geant4 model of the setup included Compton scattering and partial photon absorbtion in the scintillator and absorber, as occurring in reality. Figure 12 shows the comparison of measured and simulated attenuation curves. The model agreed with the measurements to within the experimental error. The comparison of spectra and absorption curves show that the Geant4 model suits for the interpretation of the x-ray measurements generated by the discharges.

\subsection{X-ray burst timing}

As was previously shown in the experiments described in $[14,21]$, x-rays are detected as nanosecond bursts. We provided photographic evidence that the streamer encounters act as initial accelerators in [21], as discussed in section 3.1. An example is given in figure 3 , where two detectors simultaneously record an x-ray burst at the onset of a current pulse on 


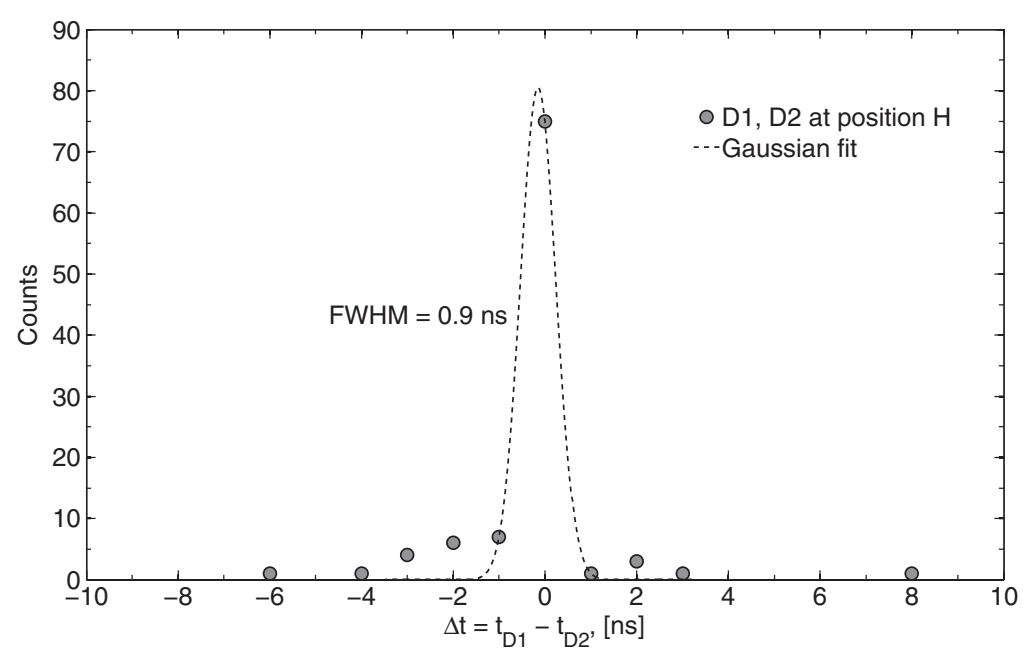

Figure 13. The delay distribution between signals recorded by two detectors placed at position $H$ next to each other. The sampling collected over $100 \mathrm{x}$-ray bursts. The dashed line is a gaussian fit with $0.9 \mathrm{~ns}$ FWHM.

the high-voltage electrode. In this case two detectors were placed at position $H$ next to each other. Figure 13 shows the delay distribution between signals accumulated over 100 $\mathrm{x}$-ray bursts detected by the two detectors. The distribution is symmetrical and peaking at zero, as it is supposed to be, giving no preference to any detector of seeing bursts earlier than the other.

The width of the peak is the measure of the photon source size-the more narrow the peak, the more localized the source. The full width at half maximum (FWHM) of $0.9 \mathrm{~ns}$ limits the average photon source volume to $27 \mathrm{~cm}$ diameter sphere. An exception to this would be the case when electrons move in the same direction as photons and produce photons at distributed points along their path.

This agrees well with the simulations. As discussed in section 3.5 , the initial electron energy converted into photon energy very rapidly. Even though some electrons can travel few meters, due to periodic collisions they lose energy reducing their radiation yield. The short $\mathrm{x}$-ray bursts also challenge the idea that the streamer front alone can generate them, since streamers propagate continuously for much longer timeshundreds of nanoseconds. This would lead to the X-ray signal broadening, which was not observed. Though a streamer probably can emit x-ray photons, taking the high electric field at its tip into account, but they do not reach the detector or stay below the detection limit.

\subsection{X-ray burst isotropy}

The calculations represented in figure 9 show that the average emission angle strongly depends on the initial electron energy. An electron starts to become relativistic for kinetic energy near its rest mass of $511 \mathrm{keV}$. When such relativistic electron hits a molecule, the Bremsstrahlung photon is emitted mostly in the forward direction. However, at lower energies the Bremsstrahlung is more isotropic. Whether the electron runs away depends on the electric field concentration between the positive and negative streamer tips during a brief moment before they collide. At this moment a run-away electron beam can be formed. The electrons are further accelerated inside as well as outside the streamer, thereby following approximately the local electric field lines, but also deviate from the lines due to collisions and hence the original beam spreads.

The spatial distribution of the x-rays during a single $\mathrm{x}$-ray burst has, to the best of our knowledge, never been investigated experimentally. Most of the observed x-rays have energies below $200 \mathrm{keV}$. The responsible electrons have larger, but comparable energies, so the Bremsstrahlung photons are rather isotropically distributed. To substantiate this claim, we conducted the following experiment. One X-ray detector was placed at position A and the other at position D; see figure 2. Over more than a hundred discharges we determined the number of simultaneous detections for both detectors. The cross-correlation between the detectors calculated over each particular X-ray burst was equal to 0.66 . In other words, if one detector absorbs $x$-rays there is a $66 \%$ chance that the second detector does so, too, within $1 \mathrm{~ns}$. In the second series of discharges, both detectors were placed next to each other at position $\mathrm{H}$. The cross-correlation for this configuration was 0.60. In case of a clear anisotropic emission, the cross-correlation should be lower when both detectors are placed apart. This was not observed.

In addition, we refer to our previous study on x-ray emission from positive discharges where the emission was also measured from different locations around the gap [14]. It was shown that at position A, which is located in opposite direction to electron propagation, the registration rate is comparable and even higher than in other locations. Here one should also take into account that the position A is much closer to the X-ray source than any others. There are two possible explanations to this: either isotropic x-ray distribution or back-scattering-when Bremsstrahlung photons emitted in opposite to electron direction. In case of back-scattering the spectrum at this detector position would be significantly softer than in others, but this was not observed.

And finally, smaller applied voltage leads to more isotropic x-rays emissions. In contrast, the $\mathrm{x}$-ray emission from lightning leaders is expected to be strongly beamed, but as 
Table 3. Registration of x-ray bursts in different positions.

\begin{tabular}{llcll}
\hline Point & $\begin{array}{l}\text { Coordinates } \\
x ; y(\mathrm{~m})\end{array}$ & $\begin{array}{l}\text { Registration } \\
\text { out of surges }\end{array}$ & $\begin{array}{l}\text { Registration } \\
P(\%)\end{array}$ & $\begin{array}{l}\text { Distance } \\
\text { from HV }(\mathrm{m})\end{array}$ \\
\hline$A^{\mathrm{a}}$ & $0.15 ;-0.13$ & $104 / 314$ & 33 & 1.22 \\
$B^{\mathrm{a}}$ & $0.35 ;-0.13$ & $32 / 120$ & 27 & 1.27 \\
$C^{\mathrm{a}}$ & $2.10 ;-0.15$ & $29 / 160$ & 18 & 2.3 \\
$D^{\mathrm{a}}$ & $1.50 ;-0.15$ & $54 / 140$ & 39 & 1.77 \\
$E^{\mathrm{b}}$ & $1.15 ;-0.3$ & $3 / 10$ & 30 & 1.8 \\
$F^{\mathrm{b}}$ & $1.50 ;-0.6$ & $25 / 60$ & 42 & 1.58 \\
$F^{\prime}$ up $^{\mathrm{b}}$ & $1.50 ;-0.6$ & $8 / 50$ & 16 & 1.58 \\
$F^{\prime}$ down & $1.50 ;-0.6$ & $4 / 50$ & 8 & 1.58 \\
$G^{\mathrm{b}}$ & $1.50 ;-2.0$ & $14 / 50$ & 28 & 1.77 \\
$H^{\mathrm{c}}$ & $2.10 ;-0.6$ & $120 / 856$ & 14 & 2.16 \\
\hline
\end{tabular}

${ }^{a}$ Series I,

${ }^{\mathrm{b}}$ Series II,

${ }^{c}$ Series III.

Note: The detection area of one detector is $11.3 \mathrm{~cm}^{2}$. The gap distance is $107 \mathrm{~cm}$. The table is reproduced from [21]

was shown in [56] it is not. Only 11 out of 21 cases in this report had a statistically significant anisotropy. We note that the authors used $\mathrm{NaI}$ detectors which are relatively slow for the fast X-ray bursts detection. So their measurements could be averaged over several individual X-ray bursts which would reduce anisotropy.

The model calculations of section 3 show us that the x-ray emission of individual streamer encounter is not isotropic, certainly not at electron energies above $0.5 \mathrm{MeV}$. But the level of anisotropy in the experiments appears to be low, likely due to relatively low initial electron and $\mathrm{x}$-ray energies. In the analysis that follows we consider the $\mathrm{x}$-ray bursts as isotropic.

\subsection{Registration rate}

In the laboratory we measured the registration rate, spectra and attenuation curves at different positions (see figure 2). We did not detect x-rays in every discharge. For example, when a single $\mathrm{LaBr}_{3}$ detector shows an $\mathrm{x}$-ray signature in 30 out of 100 discharges, we say that the registration rate at this position is $30 \%$. The registration rate contains information about the initial number of x-ray photons that can bring us to the initial number of high-energy electrons necessary to produce the $\mathrm{x}$-ray burst. The $\mathrm{x}$-ray burst are intense enough that the detector can be hit by several photons simultaneously within $1 \mathrm{~ns}$ or less, depending on the distance from the discharge. In such cases we cannot distinguish between a single $200 \mathrm{keV}$ photon and two $100 \mathrm{keV}$ photons as the photomultiplier gives an identical electrical output. But we must also consider the overlap of different bursts, in particular for energetic events with deposited energy above $1 \mathrm{MeV}$. For more information on the energy spectrum and attenuation curves we refer to our report on negative discharge in [21].

The registration rates on one $\mathrm{LaBr}_{3}$ detector were measured at different positions as indicated in figure 2 . In position $F^{\prime}$ the detectors are covered by lead tubes of $15 \mathrm{~mm}$ thickness and pointed to different parts of the gap-up and down. Their areas of view do not across. The registration rates were

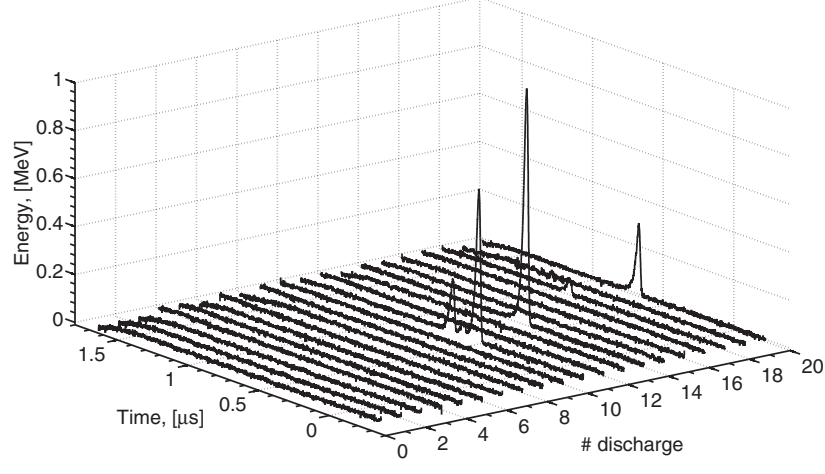

Figure 14. Oscillograms of 20 discharges measured in a row. The first nine discharges show no x-ray signature. Discharge \#10 shows a triple $\mathrm{x}$-ray burst. In total four discharges contain an x-ray signature. The registration rate in this case is equal to $20 \%$. The registration rate depends on the position of the detector.

presented in [21] and are repeated here in table 3 for the sake of completeness.

Series II was measured two months later than series I. Series III half a year later. Electrical parameters of the discharge remained constant during all series. As can be seen, when the detector is pointed to the top of the gap ( $F^{\prime}$ up) two times more events are detected than when it is pointed to the bottom $\left(F^{\prime}\right.$ down). The same behavior was observed in positive discharges, when the cathode area is twice more productive in X-rays than the anode [14]. As expected, the farthest positions $C$ and $H$ show the lowest registration rate. In general, three processes contribute to the registration rate: (1) generation rate, (2) geometrical decay and (3) attenuation by air, detector hull and EMC-cabinet. Below we consider each of them individually.

4.5.1. Registration and generation rates. Usually we detect a single $\mathrm{x}$-ray burst during one discharge, but sometimes we see up to five. The $\mathrm{x}$-ray signatures of 20 discharges generated in a row are shown in figure 14 . The time intervals between the discharges were at least $10 \mathrm{~s}$. The first nine discharges show no x-ray signature. Three x-ray bursts are detected in discharge \#10. In discharges \#13, 17 and 20 a single $x$-ray burst showed up. In total, $\mathrm{x}$-rays are detected in 4 discharges. Here we define a registration rate, which is in this particular case equals to $4 / 20=20 \%$. As mentioned already, the registration rate is calculated over discharges and not over x-ray bursts; otherwise it could assume values over $100 \%$. When a detector is moved farther away from the gap, the registration rate, as expected, drops. By analysing 950 discharges (mostly from series III, table 3 ) performed in 4 days in a row it was noticed that the ratio between single, double and triple $\mathrm{x}$-ray bursts does not vary appreciably with the detector distance. For the isotropic distribution, this implies that the number of generated X-ray bursts is dominated by the properties of the source.

Even with the fast $\mathrm{LaBr}_{3}$ detector we have to take care of possible burst overlap and pile-up. For this analysis we are mostly interested in counting the number of bursts rather than the energy per burst. We also used data from faster plastic scintillation detectors in parallel with the $\mathrm{LaBr}_{3}$. This allowed better burst separation, although burst overlaps can never be 


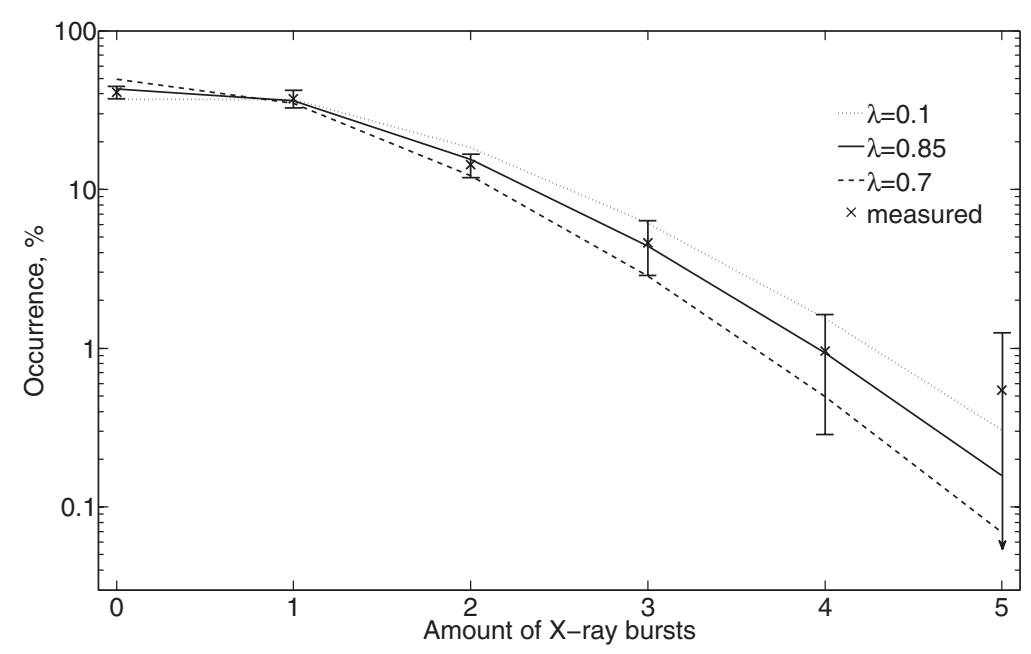

Figure 15. The number of bursts in a single discharge measured at position $F$. A Poisson distribution with $\lambda=0.85$ fits the measured data best.

fully excluded for bursts that appear seriously broadened in the $\mathrm{LaBr}_{3}$ records. Broadened here implies that the burst signal cannot be fitted to a response versus time function determined for single photons from ${ }^{137} \mathrm{Cs}$. To our advantage such bursts are rare. The probability to see $k$ bursts in a single discharge could accurately be fitted to a Poisson distribution as shown in figure 15. The best fit to the measured data is achieved with $\lambda=0.85$. With an ideal detector covering all directions and capturing all the $\mathrm{x}$-rays generated by the discharge, the probability to detect one $\mathrm{x}$-ray burst in the discharge is equal to $P(1)=36 \%$. The chance to see a double burst $-P(2)=0.15$, triple- $P(3)=0.05$, and so on. Then the probability to detect any number of $\mathrm{x}$-ray bursts in one discharge is simply their sum:

$$
P_{\max }=\sum_{k=1}^{\infty} \frac{\lambda^{k} \mathrm{e}^{-\lambda}}{k !},
$$

where $P_{\max }$ is the probability to detect x-rays with the ideal detector; $k$ the number of bursts within one discharge; $\lambda$ the Poisson distribution parameter. Equation (8) results in $P_{\max }=0.57$ - the maximal chance that any number of x-rays will be detected in a discharge. As we pointed out above, the most likely candidate for the x-ray source is a streamer encounter where positive corona of a bipolar pilot system meets negative streamers emanated from the high-voltage electrode. It was shown in [15] that such encounters happen at later stages of pre-breakdown phenomenon and are associated with x-ray bursts [21]. But if, for any reason they do not meet or the electric field is not intense enough over sufficiently long distance and period, no x-rays will be created. There is no fundamental reason why each single discharge in our laboratory would generate $\mathrm{x}$-rays. Note that the low-energy limit of our x-ray detectors is $30 \mathrm{keV}$. Bursts with lower photon energies can appear, but are less likely to be detected. The parameter $\lambda$ is a characteristic of our lab environment in general and the high-voltage electrode shape in particular. It was demonstrated in [14] how the number of x-rays can be increased by changing the number of negative streamers in positive discharges.
4.5.2. Geometrical decay. In [21] we showed that the x-ray source is located near the HV electrode. It is also clear from measurements with collimators at position $F^{\prime}$ (up and down) in table 3 . If all source locations are distributed equally on a ring with fixed radius around the axis, the average source location is on the axis. Since we also assume isotropic x-ray bursts, the geometrical decay influences the registration rate, especially at large distances. As can be seen from table 3, the registration rate decreases with the distance from the gap. For simplicity we count the distance from the high voltage electrode tip. Consider an X-ray burst that emits $n_{\mathrm{ph}}$ photons in $4 \pi$ sterad. Up to some distance $r_{s}$ the flux through the detector equals to one or more photons per burst. At these distances the registration rate is only limited by the generation rate, which is $57 \%$ $\left(P_{\max }\right)$. In other words, the detector registers each generated burst. At distances further than $r_{s}$, the registration rate decays according to inverse square law starting from its maximum:

$$
P(r) \sim \frac{1}{r^{2}} \cdot P_{\max } \text { for } r>r_{s} .
$$

The photon flux through the detector at distance $r$ can be estimated by the following equation:

$$
F(r)=\frac{n_{\mathrm{ph}}}{4 \pi} \cdot \frac{1}{r^{2}} \cdot \pi r_{d}^{2},
$$

where $F$-number of photons per detector area at distance $r$, $r_{d}$-the detector scintillator radius, $n_{\mathrm{ph}}$ - total number of initial photons emitted in $4 \pi$. The requirement $r_{d} \ll r$ is satisfied. For simplicity here we just use geometrical scintillator radius instead of effective detection area which is energy dependent and will be considered below.

Then the registration rate can be generalized:

$$
P(r)=\left\{\begin{array}{cc}
F(r) \cdot P_{\max }, & r>r_{s} \\
P_{\max }, & r \leqslant r_{s} .
\end{array}\right.
$$

Now the registration rate can be calculated for different initial photon numbers $n_{\mathrm{ph}}$ and compared to the measured values summarized in table 3 . Figure 16 shows the registration rate at different locations. The solid and dashed lines are the 


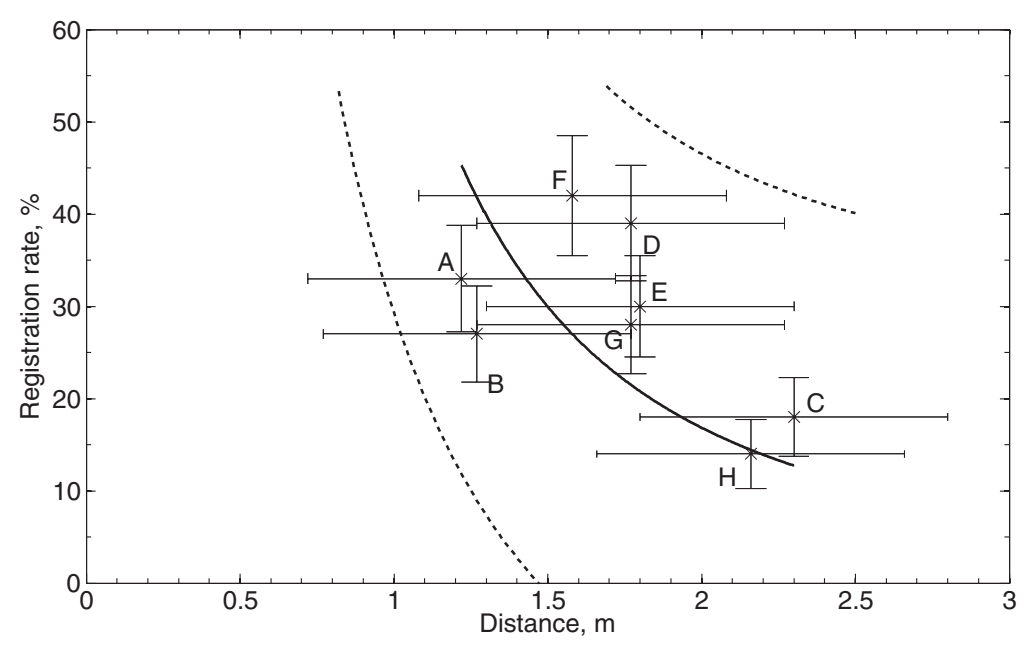

Figure 16. The registration rate versus distance from the $\mathrm{x}$-ray source to the detector. The lines show fit by $1 / r^{2}$ with $95 \%$ confidence bounds and $0.66 \mathrm{R}$-squared. Measured registration rates at different locations are also indicated. Estimated number of photons above $30 \mathrm{keV}$ in an average x-ray burst is $n_{\mathrm{ph}}=(7.5 \pm 2.7) \cdot 10^{3}$.

fitted function $P(r)$ with $95 \%$ confidence bounds. The large horizontal error bars are due to the uncertainty in the x-ray source location (see figure 8 in [15]). The initial number of photons $n_{\mathrm{ph}}$ needed for X-ray burst and the parameter $r_{s}$ can now be derived from the fit and equation (10); we obtained $n_{\mathrm{ph}}=(7.5 \pm 2.7) \cdot 10^{3}$ in $4 \pi$ sterad and $r_{s}=1.6 \pm 0.3 \mathrm{~m}$. This $n_{\mathrm{ph}}$ is a minimum necessary brightness averaged over all discharges, where we did not account for possible pile-up. A better approach includes the energy information and is shown below.

\subsection{Characterisation of $x$-ray bursts through measurements and simulation}

We simulate the X-ray bursts near the HV electrode in the Monte-Carlo approach using the Geant 4 model of the detector. In order to avoid the problem of multi-photon detection and burst pile-up, we now only consider those bursts for which the measured signal can be fitted to a single photon response. With the model of section 3 we could in principle convert the number of electrons to photons. In reality individual bursts may have their individual electron energy spectrum. However, no presently known model can cope with the complexity of the discharge and streamer encounters. As a result we still have to assume a spectral distribution for the photons as in [21]. We choose an exponential $\mathrm{e}^{-\epsilon / \epsilon_{c}}$ photon energy distribution. This choice might appear somewhat arbitrary, as it does not naturally limit the photon energy in relation to the maximum voltage. We therefore limited the single photon energy to values between $30 \mathrm{keV}$ and $1 \mathrm{MeV}$. Again we assume isotropy. By varying the number of initial photons and the characteristic energy $\epsilon_{c}$ we obtained parameters that simultaneously fit the spectrum and the attenuation curves measured and published before in [21]. For the detector at position $D$ the results of the simulation are shown in figure 17, where we plotted surfaces of registration rates as a function of $\epsilon_{c}$ and $n_{\mathrm{ph}}$, with the lead attenuator thickness as a parameter. The best fitting set $\epsilon_{c}=160 \mathrm{keV}$ and $n_{\mathrm{ph}}=6 \cdot 10^{4}$ is indicated by the dashed lines. Figure 18 shows different cross sections of figure 17 with error-bars derived from the Monte-Carlo process.

In figure 19 we compare the simulated and measured pseudo-spectra without absorber, for detector position $\mathrm{H}$, where we split the energy range in bins of $50 \mathrm{keV}$. In order to allow the comparison with the measurements, we normalized the total number of $\mathrm{x}$-ray photons i.e. occurrences to 1 for the calculated and measured data. Up to $0.5 \mathrm{MeV}$ the agreement is good. This is a remarkably large energy range, three times the characteristic energy $\epsilon_{c}$ and a spectral intensity range of about one decade. Please note that the spectrum has not been fitted directly, but came out of the fit shown in figure 17. The dotted-dashed line represents the exponential distribution for single photon detection. The Geant 4 model implicitly includes the photon pile-up; in position $H$ on the average 1.2 photons simultaneously hit the detector for $n_{\mathrm{ph}}=6 \cdot 10^{4}$. With a larger $n_{\mathrm{ph}}$ the number of multiphoton detections increases above $0.5 \mathrm{MeV}$ at the expense of the lower energy number, and the spectrum fits less well. At $0.5 \mathrm{MeV}$ the assumption of an isotropic photon distribution becomes less valid, and the burst pile-up becomes important in the measurements. This is a quite probable explanation in view of the maximum applied voltage of $1 \mathrm{MV}$ and the sharp drop in photon production at higher energies shown in figure 10.

\section{Discussion and conclusion}

In previous articles $[14,15,21]$ we have experimentally investigated the development of long laboratory sparks, we have characterized their evolution in space and time, together with current and voltage, we have identified at which stage they emit $\mathrm{X}$-ray bursts, and we have measured these x-rays in space and time.

In the present article, we have focussed on modeling the $\mathrm{x}$-ray generation in the observed discharges, on x-ray propagation and detection and on comparison with experimental data, concentrating on negative discharges - though the analysis for positive ones follows essentially along the same lines. 


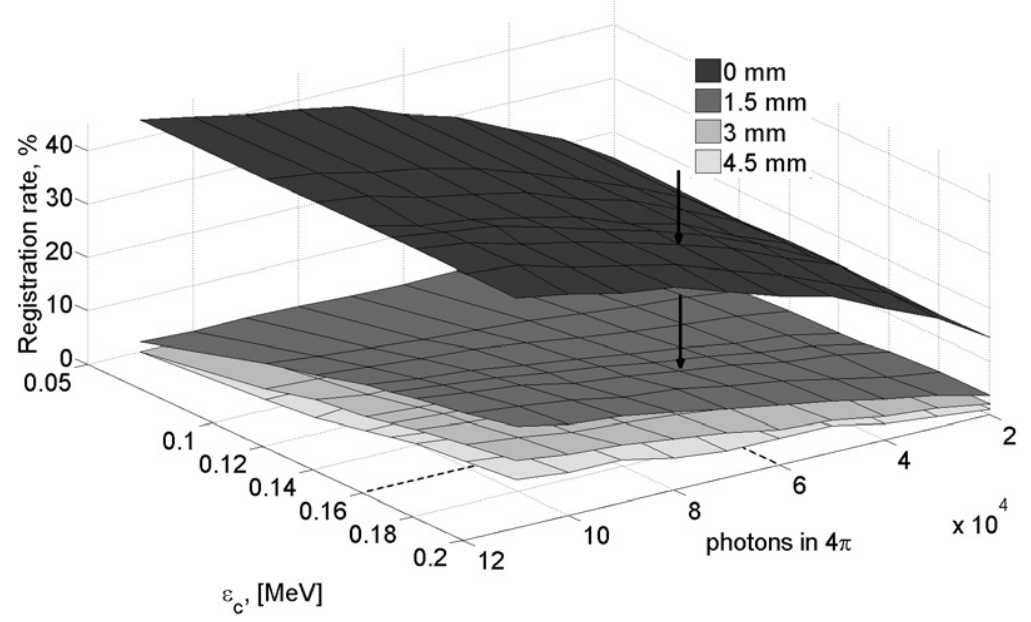

Figure 17. The registration rate versus source intensity $n_{\mathrm{ph}}$ versus characteristic photon energy $\epsilon_{c}$ calculated at position $D$. Four planes are simulated for different attenuator thicknesses in front of the detector. Points on the planes marked by arrows correspond to the best fit to the measured attenuation curves.
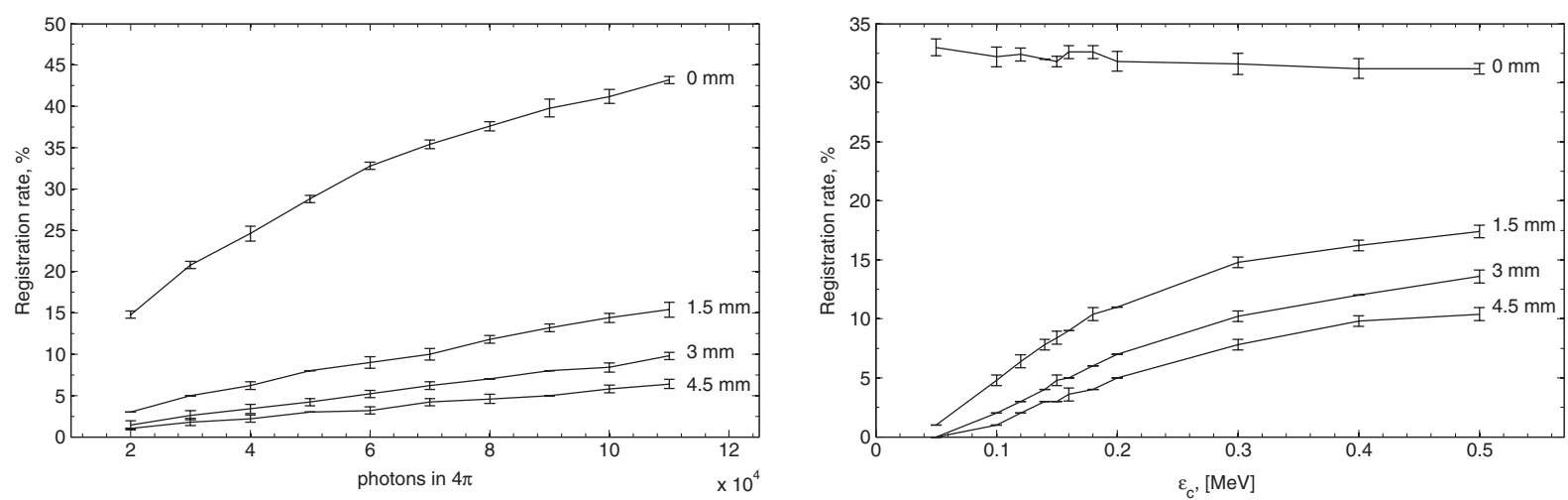

Figure 18. (Left) The registration rate versus source intensity for different attenuator thicknesses. The characteristic energy $\epsilon_{c}$ is fixed to $160 \mathrm{keV}$. The registration rates at $6 \cdot 10^{4}$ photons coincide with those measured at the lab. (Right) The registration rate versus characteristic photon energy $\epsilon_{c}$ for different attenuator thicknesses. The initial number of photons is fixed to $6 \cdot 10^{4}$ in $4 \pi$ sterad. At $\epsilon_{c}=160 \mathrm{keV}$ the registration rates coincide with those measured in the lab.

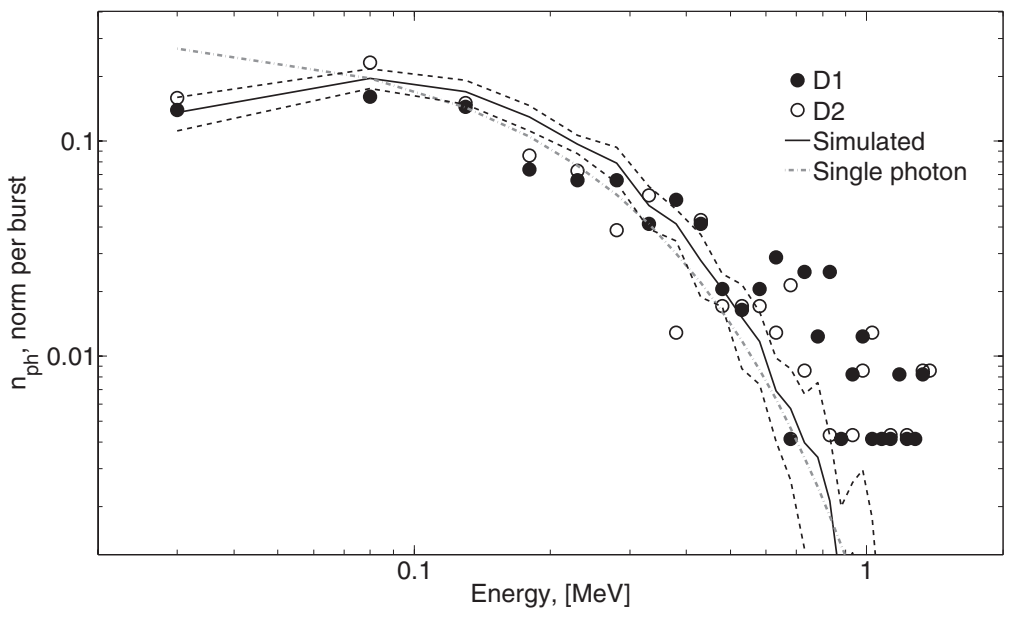

Figure 19. Simulated and measured spectra measured by detectors D1 and D2 spectra at position $H$. Bin width is $50 \mathrm{keV}$. The initial number of photons with energy over $30 \mathrm{keV}$ is $6 \cdot 10^{4}$ over $4 \pi$ sterad and the characteristic energy $\epsilon_{c}$ is equal to $160 \mathrm{keV}$. The dot-dash line represents the exponential distribution for single photon detection.

Our photographs indicate that $\mathrm{x}$-ray bursts occur in our experiments when two growing discharge channels of opposite polarity encounter each other; this happens quite often near the high-voltage electrode. Immediately before the merging, the very high electric field between the two approaching 'conductors' can let the electrons run away. But only in $60 \%$ of 
the discharges we observed x-rays, in spite of many streamer encounters. This indicates that $\mathrm{x}$-ray bursts from streamer encounters are a rare phenomenon.

As reliable models for discharge encounters and associated electron acceleration within complex discharge trees are not available, we have assumed that the discharge encounter occurs near the cathode and emits an electron beam with energies between 30 and $100 \mathrm{keV}$. This electron beam is further accelerated in the electric field of the discharge for which we provide a simple approximation. Just outside the discharge zone at about $1 \mathrm{~m}$ distance from the cathode, we estimate that the electrons reach an energy maximum of 500-600 keV. This remains true when the streamer encounter and electron acceleration occurs at some distance from the cathode, be it that the maximal attained energy is smaller. In this region the generation of hard $\mathrm{x}$-rays through Bremsstrahlung radiation of the electrons is the most likely, because the electron energy is maximal.

Energy and path of these electrons in the electric field outside the discharge are modeled with a Monte Carlo model. As the problem is linear, it is sufficient to consider unidirectional and monoenergetic electron beams, and to compose general solutions by superposition. We have first studied electron beams within the radially directed discharge field to illustrate their spreading through scattering and field as well as their energy losses.

Then we have studied the generation of x-rays by monoenergetic electron beams with energies of $100 \mathrm{keV}-1 \mathrm{MeV}$, and we have characterized the $\mathrm{x}$-ray distribution in space and energy. Electron beams with an initial energy of $1 \mathrm{MeV}$ produce 18 times as many photons with energy above $30 \mathrm{keV}$ than electron beams with an initial energy of $200 \mathrm{keV}$; all of these photons are produced within few ns. For the photon number per electron as a function of electron energy, a fit formula to the numerical data is presented.

Another fit to the data of Monte Carlo simulations relates the opening angle of an x-ray beam to the electron energy. While photons produced by $\mathrm{MeV}$ electrons are emitted mostly in the forward direction relative to the direction of the original electron beam, photons produced by lower energy electrons are emitted more isotropically. Additionally Compton scattering changes the direction of low-energy photons and widens the photon beam. So in the evaluation of the experimental data we will assume the photon burst to be isotropic in space, as the following effects add up: the electrons are emitted from the streamer encounter region with slightly diverging directions. Then they spread further due to scattering and due to the radial electric field. Finally the photons are emitted with some opening angle even from some unidirectional beam.

Another observation is that photons with energies between $\approx 100 \mathrm{eV}$ and $\approx 10 \mathrm{keV}$ are largely lost before reaching the detector due to photoionization where a photon is absorbed by an air molecule and an electron is emitted, while photons with energy above $30 \mathrm{keV}$ stay essentially unattenuated when crossing $2 \mathrm{~m}$ of air. This provides a lower cut-off for the spectrum of observed photons. On the other hand, the high-energy tail of the photon distribution can be fitted quite well by the Bethe-Heitler equation. A characteristic energy of the photon energy distribution in the range of $100 \mathrm{keV}$ thus follows naturally from these considerations, in agreement with the experiments.

When evaluating our experimental data, we assume the $\mathrm{x}$-ray bursts to be isotropic when they reach the detectors. The number of x-ray bursts in a single discharge follows a Poisson distribution with mean value $\lambda=0.85$. We found three main process contributing to the $\mathrm{x}$-ray detection. These are: generation rate, geometrical decay and attenuation. By creating a precise Geant 4 model of $\mathrm{LaBr}_{3}$ detector we simulate its response. It is consistent with the measurements.

The simulations indicate that also electrons might arrive at the EMC cabinet, pass through the aluminum window and enter the detector. Still we have reasons to support the notion that the recorded signals are mainly if not solely due to x-rays: the large energies recorded, and the attenuation curves that agree reasonably well with x-rays while electrons are much stronger absorbed in lead.

The $\mathrm{x}$-rays arrive at adjacent detectors with a spread on the nanosecond time scale, and there are only few ones per detector. This indicates that the x-ray burst passes the detectors within nanoseconds, which gives a strong constraint in the duration of the initial acceleration mechanism.

A simple geometrical model for the number of $\mathrm{x}$-ray occurrences as a function of detector distance required about $7 \cdot 10^{3}$ initial photons when averaged over all discharges (see figure 16). A detailed Geant4 simulation showed that $6 \cdot 10^{4}$ photons are needed per $\mathrm{x}$-ray burst. The exponential photon energy distribution $\mathrm{e}^{-\epsilon / \epsilon_{c}}$ between $30 \mathrm{keV}$ and $1 \mathrm{MeV}$ with $\epsilon_{c}=160 \mathrm{keV}$ describes both the absorption by lead plates and the spectrum correctly.

With $6 \cdot 10^{4}$ photons and $10^{2}$ electrons at $200 \mathrm{keV}$ per photon (see equation (5)), one needs a charge of approximately $1 \mathrm{pC}$ to generate the observed x-rays. In figure 3 the current starts to rise at the begin of the fourth streamer burst in an approximately linear way with $1 \mathrm{~A} \mathrm{~ns}^{-1}$ in the first $100 \mathrm{~ns}$. During $1 \mathrm{~ns}$ the charge injected the streamer cloud is then $0.5 \mathrm{nC}$, at factor of 500 larger than the charge needed for the $\mathrm{X}$-rays. The total charge inside the streamer cloud is then of the order of several tens of $\mu \mathrm{C}$. Only a minute fraction of the electrons runs away.

Although this study is based on negative discharges the same thoughts are applicable for x-rays from positive discharges [14]. The registration rate in positive discharges is about twice as much as in negative ones. This is reflected to higher Poisson parameter $\lambda$. In other words, the generation rate is higher for positive discharges than for negative ones in our setup.

New experiments are in preparation that focus on the open questions.

\section{Acknowledgments}

Authors acknowledge funding by STW project 10757 in The Netherlands. P Kochkin also acknowledges the European Research Council and the European Union's Seventh Framework Programme (FP7/2007-2013)/ERC grant 
agreement n. 320839 and the Research Council of Norway under contracts 208028/F50, and 223252/F50 (CoE).

\section{References}

[1] Wilson C T R 1924 Proc. Phys. Soc. London 37 32D

[2] Stankevich Y and Kalinin V 1967 Sov. Phys._Dokl. 12 1042-3

[3] Noggle R C, Krider E P and Wayland J R 1968 J. Appl. Phys. 39 4746-8

[4] Dwyer J R, Rassoul H K and Saleh Z 2005 Geophys. Res. Lett. 32 L20809

[5] Kostyrya I D, Tarasenko V F, Tkachev A N and Yakovlenko S I 2006 Technol. Phys. 76 64-9

[6] Dwyer J R, Saleh Z, Rassoul H K, Concha D, Rahman M, Cooray V, Jerauld J, Uman M A and Rakov V A 2008 J. Geophys. Res. 113 D23207

[7] Nguyen C V, van Deursen A P J and Ebert U 2008 J. Phys. D: Appl. Phys. 41234012

[8] Rahman M, Cooray V, Ahmad N A, Nyberg J, Rakov V A and Sharma S 2008 Geophys. Res. Lett. 35 L06805

[9] Rep'ev A G and Repin P B 2008 Technol. Phys. 53 73-80

[10] Nguyen C V, van Deursen A P J, van Heesch E J M, Winands G J J and Pemen A J M 2010 J. Phys. D: Appl. Phys. $\mathbf{4 3} 025202$

[11] March V and Montanyà J 2010 Geophys. Res. Lett. 37 L19801

[12] March V and Montanyà J 2011 Geophys. Res. Lett. 38 L04803

[13] Tao S, Zhang C, Niu Z, Yan P, Tarasenko V F, Baksht E K, Burahenko A G and Shut'ko Y V 2011 Appl. Phys. Lett. 98021506

[14] Kochkin P O, Nguyen C V, van Deursen A P J and Ebert U 2012 J. Phys.: D Appl. Phys. 45425202

[15] Kochkin P O, van Deursen A P J and Ebert U 2014 J. Phys. D: Appl. Phys. 47145203

[16] Kochkin P et al 2015 J. Phys. D: Appl. Phys. 48425202

[17] Tarasenko V F, Baksht E K, Burachenko A G, Kostyrya I D, Lomaev M I and Rybka D V 2008 Plasma Devices Oper. 16 267-98

[18] Dwyer J R 2004 Geophys. Res. Lett. 31 L12102

[19] Berger M J, Coursey J S, Zucker M A and Chang J 1998 NIST, Physical Measurement Laboratory retrieved December 21, 2015 http://physics.nist.gov/PhysRefData/ Star/Text/ESTAR.html

[20] Cooray V, Arevalo L, Rahman M, Dwyer J and Rassoul H 2009 J. Atmos. Sol.-Terr. Phys. 71 1890-8

[21] Kochkin P O, van Deursen A P J and Ebert U 2015 J. Phys. D: Appl. Phys. 48025205

[22] Petersen D A and Beasley W H 2013 J. Geophys. Res.: Atmos. $11812-110$

[23] Köhn C and Ebert U 2015 J. Geophys. Res. Atmos. 120 1620-35

[24] Luque A, Ratushnaya V and Ebert U 2008 J. Phys. D: Appl. Phys. 41234005

[25] Briels T M P, Kos J, Winands G J J, van Veldhuizen E M and Ebert U 2008 J. Phys. D: Appl. Phys. 41234004

[26] Gallimberti I, Bacchiega G, Bondiou-Clergerie A and Lalande P 2002 C. R. Phys. 3 1335-59
[27] Nijdam S, Takahashi E, Teunissen J and Ebert U 2014 New J. Phys. 16103038

[28] Nijdam S, Takahashi E, Markosyan A and Ebert U 2014 Plasma Sources Sci. Technol. 23025008

[29] Moss G D, Pasko V P, Liu N and Veronis G 2006 J. Geophys. Res. 111 A02307

[30] Li C, Ebert U and Hundsdorfer W 2009 J. Phys. D: Appl. Phys. 42202003

[31] Chanrion O and Neubert T 2010 J. Geophys. Res.: Space Phys. 115 A6

[32] Celestin S and Pasko V P 2011 J. Geophys. Res. 116 A 03315

[33] Köhn C and Ebert U 2014 Plasma Sour. Sci. Technol. 23045001

[34] Köhn C, Ebert U and Mangiarotti A 2014 J. Phys. D: Appl. Phys. 47252001

[35] Electron scattering database LXCat www.lxcat.laplace.univtlse.fr

[36] Jacob J 1973 Phys. Rev. A 8 226-35

[37] Phelps A V and Pitchford L C 1985 Phys. Rev. A 31 2932-49

[38] Phelps A V 1985 Jila information center report no. 28 Technical Report University of Colorado

[39] Fiala A, Pitchford L C and Boeuf J P 1994 Phys. Rev. E 495607

[40] Vahedi V and Surendra M 1995 Comput. Phys. Commun. 87 179-98

[41] Lawton S A and Phelps A V 1978 J. Chem. Phys. 69 1055-68

[42] Kim Y K 2000 Phys. Rev. A 62052710

[43] Bethe H A and Heitler W 1934 Proc. Phys. Soc. 146 83-112

[44] Heitler W 1944 The Quantum Theory of Radiation (Oxford: Oxford University Press)

[45] Köhn C and Ebert U 2014 Atmos. Res. 135 432-65

[46] Luque A and Gordillo-Vázquez F J 2012 Nat. Geosci. 5 22-5

[47] Pancheshnyi S, Eismann B, Hagelaar G J M and Pitchford L C 2008 Computer code zdplaskin university of Toulouse, LAPLACE, CNRS-UPS-INP, Toulouse, France www. zdplaskin.laplace.univ-tlse.fr

[48] Chanrion O and Neubert T 2008 J. Comput. Phys. $2277222-45$

[49] Li C, Teunissen J, Nool M, Hundsdorfer W and Ebert U 2012 Plasma Sources Sci. Technol. 21055019

[50] Celestin S and Pasko V P 2010 J. Phys. D: Appl. Phys. 43315206

[51] Photon and electron interaction data (retrieved December 21 2015) 1997 www-nds.iaea.org/epdl97/

[52] Greiner W and Reinhardt J 1995 Quantenelektrodynamik (Berlin: Verlag)

[53] Peskin M E and Schroeder D V 1995 An Introduction to Quantum Field Theory (New York: Westview Press)

[54] Shardanand and Rao A D P 1977 Absolute rayleigh scattering cross sections of gases and freons of stratospheric interest in the visible and ultraviolet regions Technical Report Wallops Flight Center

[55] Nguyen C V 2012 Experimental study on hard radiation from long laboratory spark discharges in air PhD Thesis Eindhoven University of Technology http://alexandria.tue. nl/extra2/731153.pdf

[56] Schaal M M, Dwyer J R, Rassoul H K, Hill J D, Jordan D M and Uman M A 2013 J. Geophys. Res.: Atmos. $11811712-26$ 